\title{
Advances in Angioscopic Imaging of Vascular Disease
}

\author{
Yasumi Uchida ${ }^{1,2,3^{*}}$, Yasuto Uchida ${ }^{4}$ \\ ${ }^{1}$ Japanese Foundation for Cardiovascular Research, Funabashi, Japan \\ ${ }^{2}$ Public Trust Japanese Cardiovascular Research Fund for Young Investigators, Tokyo, Japan \\ ${ }^{3}$ Department of Cardiology, Jikei University School of Medicine, Tokyo, Japan \\ ${ }^{4}$ Department of Cardiology, Toho University Medical Center, Tokyo, Japan \\ Email: *uchiy@ta2.so-net.ne.jp
}

Received October 13, 2012; revised November 16, 2012; accepted November 29, 2012

\begin{abstract}
Percutaneous angioscopy, using high resolution fiberoptic imaging, allows direct and two-dimensional visualization of the vascular interior, thereby enabling macroscopic pathological diagnosis. Percutaneous angioscopy has revealed that the vascular luminal surface exhibits various colors and morphologies characteristic of different vascular diseases. This imaging technique is used for evaluation of the severity of vascular diseases, staging of atherosclerosis, analysis of thrombus composition, evaluation of interventional and surgical therapies, and for guidance of intravascular interventions such as angioplasty, venous valvuloplasty and aortic stentgrafting. Recently, dye-image angioscopy has been used clinically for analyses of thrombus composition, endothelial damage and plaque composition. Intravascular microscopy was also developed for cellular imaging of vascular disease. Furthermore, fluorescent angioscopy was developed for molecular imaging of substances comprising atherosclerotic plaques. In this article, we describe the history of the development of angioscopy, angioscopic systems and techniques, angioscopic changes associated with vascular diseases, angioscope-guided intravascular therapies, and evaluation of intravascular and surgical therapies. Angioscopic pictures, except those of the coronary arteries, have rarely been published in the literature, so we have included many representative angioscopic pictures obtained by the authors in this article.
\end{abstract}

Keywords: Angioscope-Guided Intravascular Interventions; Evaluation of Interventional and Surgical Therapies; Percutaneous Angioscopy

\section{Introduction}

Direct observation of changes in the vascular wall was previously beyond the scope of any available imaging modalities.

Percutaneous angioscopy (AS) is a high resolution fiberoptic imaging technique which enables direct visualization of the vascular wall from within, thereby enabling macroscopic pathological diagnosis of vascular diseases. This imaging technique is now clinically employed not only in the diagnosis of vascular diseases, but also for the evaluation of interventional and surgical therapies and guidance of intravascular interventions.

This article presents the past, present and future prospects of this promising imaging technique.

\section{Overview of Literature on Angioscopy (AS)}

Intravascular observations using a rigid endoscope in animals were performed by Allen et al. in 1922 [1] and by Harken et al. in 1943 [2].

\footnotetext{
"Corresponding author.
}

In 1956, Sakakibara et al. employed a rigid endoscope to examine atrial septal defects during open-heart surgery [3]. They also examined aortic valves using the same endoscope in 1958 [4].

Many years later, thin and flexible angioscopes were developed for percutaneous observation of not only coronary and peripheral vessels, but also the great vessels, and many clinicians have engaged in AS imaging of the cardiovascular system.

Peripheral vascular changes in animals were observed by Sugie (1969) [5], Litvack (1985) [6], Uchida (1988) [7], and Buckmaster (1995) [8], among others.

Human coronary arteries were examined by Spears (1985) [9], Uchida (1985) [10], Sanborn (1986) [11], Hombach (1986) [12], Sherman (1986) [13], Uchida (1989) [14], Nakamura (1992) [15], Uchida (1990) [16], Hombach (1992) [17], among others.

Peripheral arteries in patients wereexamined by Uchida (1992) [18], Drobinski (1993) [19], Kollar (1997) [20], Trubel (1999) [21], among others.

The aorta was examined by Uchida (1995) [22], Hill (1995) [23] Tokuhiro (2000) [24], Tsagakis (2010) [25], 
Aono (2007) [26], among others.

Peripheral veins were examined by Hoshino (1993) [27], Yamaki (2002) [28], Nishibe (2007) [29], Konami (2010) [30], among others.

The caval veins were examined by Uchida (1995) [31], but not by others.

The pulmonary arteries were examined by Shure (1985) [32], Uchida $(1995,2010)[33,34]$, and others.

The cardiac chambers and valves were examined by Uchida $(1991,2001)$ [35,36].

Cellular AS, dye-staining AS and molecular AS were developed and used clinically by Uchida (1995, 2010, 2012) [37-42].

\section{Developmental History of Our AS System}

Difficulties in producing a thin endoscope that can safely be introduced percutaneously into the vessels, and equipment that can displace blood, meant that about 29 years elapsed before Uchida and his coworkers successfully performed percutaneous AS in patients [5,6]. Although this new modality of diagnosis is now performed routinely in a number of institutions, it has yet to be adopted on a global scale.

In 1975, a 9F fiberscope was developed in collaboration with Olympus Corporation, Tokyo. This AS was introduced through an $11 \mathrm{~F}$ hard tipped guiding catheter into a canine left ventricle, but the procedure was abandoned due to marked damage to the endocardial surface. In 1976, a 10F balloon tipped guiding catheter was developed. This catheter allowed the passage of a $6 \mathrm{~F}$ fiberscope. However, this AS also had to be abandoned because the balloon became frosty during use due to the temperature difference between the saline used for balloon dilatation and the blood in the ventricle. In the same year, a fiberscope was devised with a balloon at its tip. This fiberscope had a central lumen through which warmed saline at body temperature could be infused to dilate the balloon. The balloon was pushed against the vascular luminal surface to observe changes through the dilated balloon. However, introduction of this fiberscope into the vessel was very difficult because a guide wire could not be used, and if used in combination with a guiding catheter, a large bore catheter had to be used to allow the fiberscope to pass through. This fiberscope was not used clinically. In 1983, a 9F balloon guide catheter was devised in collaboration with the Clinical Supply Company, Gifu, Japan. When inflated with $\mathrm{CO}_{2}$, the balloon protruded distally to the shaft tip to form a dead space between the target and the balloon, at the same time preventing damage to the target tissue by the shaft tip. In combination with a $4.8 \mathrm{~F}$ fiberscope, this balloon catheter enabled percutaneous transluminal observation of vessels. This AS system is now routinely used clinically for ob- servation, not only of peripheral vessels, but also great vessels such as pulmonary arteries, caval veins and aorta.

Angioscopy of aorta and venous system was named as aortoscopy and phleboscopy, respectively $[22,31]$.

\section{Our AS System}

Our AS system comprises a light source, 1.7- 4.5F fiberscope, 6 - 9F guiding balloon catheter, Intensified Chilled Coupled Device (ICCD) camera, camera controller, image divider, DVD recorder and television monitor.

Usually, a 4.5F fiberscope and 9F balloon guide catheter are used for observation of large diameter vessels. The 4.5F fiberscope (AF 14, Olympus Corporation, Tokyo) contains 3000 glass fibers for image guidance and 300 glass fibers for light guidance. The fiberscope is passed through a 9F balloon guide catheter (Clinical Supply Company, Gifu, Japan). The balloon is inflated with $\mathrm{CO}_{2}$. The catheter has a Y connecter at the proximal end: one channel for fiberscope insertion and another for saline flushing. The white balance of the AS is adjusted using white gauze immersed in saline solution as the white color (Figure 1).

Details of various types of AS systems have been described elsewhere [43].

\section{AS Procedures (Figure 2)}

\subsection{Peripheral Artery}

The guiding balloon catheter is introduced antegradely through a sheath into either the superficial or deep femoral artery. Next, the AS is introduced via the guiding catheter to place the AS tip at the distal end of the guiding catheter. The balloon is then gently inflated with $\mathrm{CO}_{2}$ to occlude the lumen to stop blood flow. Then, the AS is advanced or pulled back for observations during saline infusion at $5 \mathrm{~mL} / \mathrm{min}$ manually or using a power injector (Figure 2(c)). In cases of observation of a branch distal to the popliteal artery, a 5F AS for coronary use (the fiberscope is incorporated into a $5 \mathrm{~F}$ balloon catheter) is employed) (Figure 1(c)).

When a severe stenosis exists at the superficial femoral arterial orifice, it is sometimes necessary to expose the femoral artery surgically, or use a contralateral approach.

For observation of the iliac artery, a guiding balloon catheter is advanced from the contralateral femoral and iliac artery through the bifurcation into the targeted iliac artery (Figure 1(a)), or retrogradely from the ipsilateral femoral artery into the iliac artery (Figure 1(b)). The former approach is sometimes difficult when the angle between the iliac arteries is sharp.

\subsection{Peripheral Veins}

The balloon catheter should not be introduced into adistal 
vein because this maneuver may damage the venous valves. The common and external iliac veins can be examined by introducing the balloon catheter retrogradely from the ipsilateral femoral vein. Observation of internal iliac vein is limited to its outlet because the angioscope may damage valves.
For observation of more distal branches of the femoral veins, a $6 \mathrm{~F}$ balloon catheter is introduced through a vein located near the ankle and a $1.7 \mathrm{~F}$ fiberscope is introduced into the balloon catheter. This maneuver enables successsive observation of multiple venous valves located in distal veins up to the femoral vein.
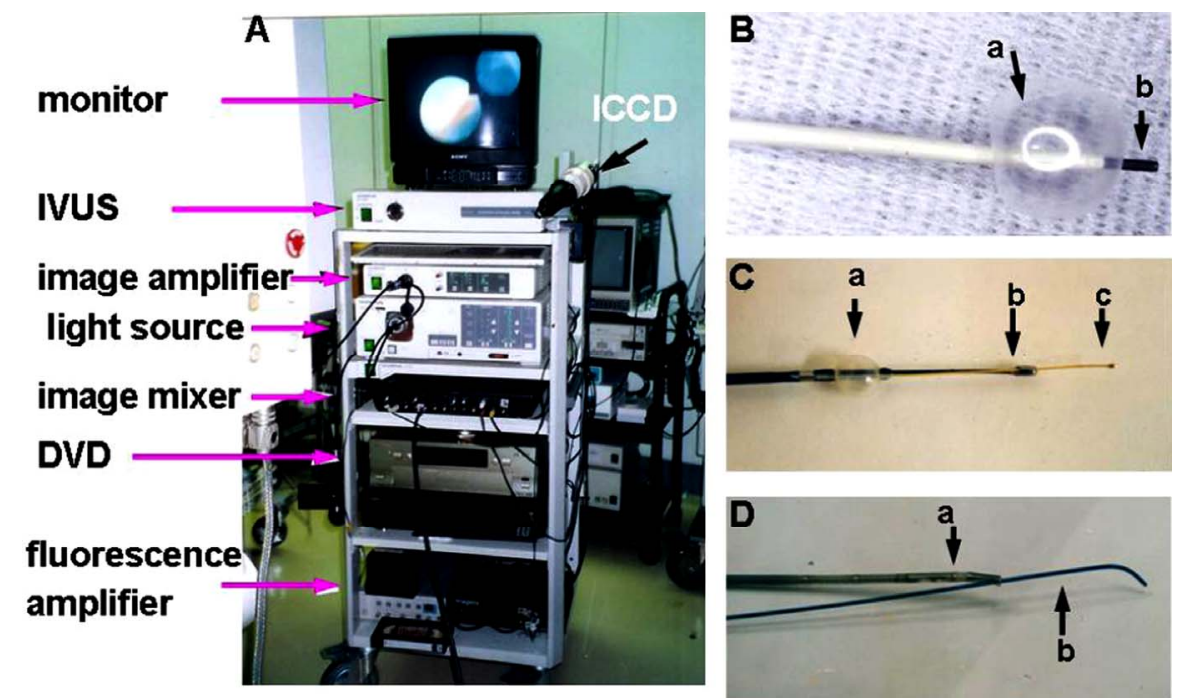

A: The system comprises a light source, image amplifiers for color and fluorescent AS and IVUS, image mixer, recorder, and monitor. ICCD: intensified chilled coupled device; B: An AS for large vessel and cardiac use. (a) 9F guiding balloon catheter; (b) 5F-fiberscope; (c) a 5F AS for small vessel use. C: (a) Guiding balloon catheter; (b) Fiberscope; (c) Guide wire; D: IVUS probe for large vessel use. (a) Probe; (b) Guidewire. Cited from reference 43 with permission.

Figure 1. Angioscopy (AS) and intravascular ultrasonography (IVUS) system used in our catheterization laboratory.
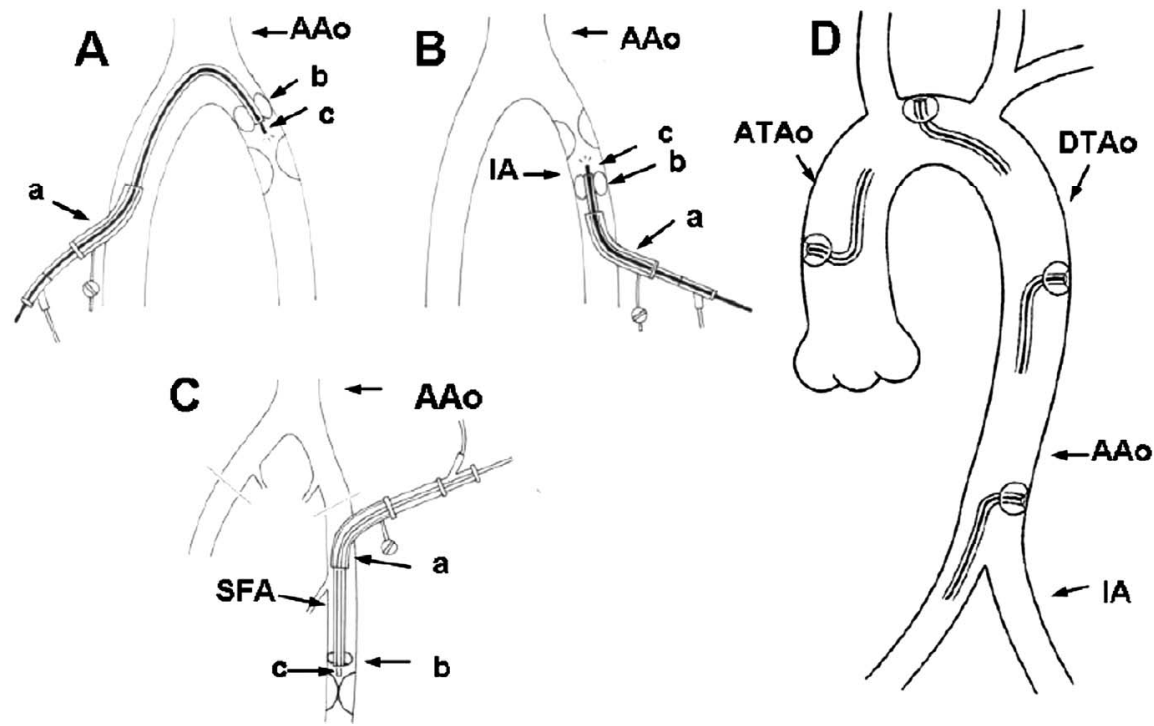

A: Iliac artery examination introducing an AS incorporated in a guiding balloon catheter through the contralateral femoral artery and across the bifurcation of common iliac artery into the ipsilateral iliac artery. a, b and $\mathrm{c}$ in this and other panels: introducer, guiding balloon catheter and fiberscope, respectively. AAo: abdominal aorta; B: Observation of iliac artery introducing an AS retrogradely through the ipsilateral femoral artery. IA: iliac artery; C: Examination of the femoral artery and its branches introducing an AS antegradely; D: Examination of the aorta. The guiding balloon catheter tip is pre-shaped depending on the target segment of the aorta to be imaged. ATAo: ascending thoracic aorta. DTAo: descending thoracic aorta. Cited from references 46 and 48 with permission.

Figure 2. Schematic representation of AS procedure. 


\subsection{Aorta}

Because the aortic diameter is larger than that of the balloon of the guiding catheter, it is impossible to stop aortic flow using the present AS system.

Following aortography and intravascular ultrasonography (IVUS), the balloon catheter is introduced retrogradely using a guide wire through a femoral artery, and an AS is introduced into the aorta, then the balloon is inflated and gently placed against the aortic luminal surface. Since the balloon protrudes $5 \mathrm{~mm}$ ahead of the catheter tip, the distance between the fiberscope tip and the aortic luminal surface is maintained at almost $5 \mathrm{~mm}$ $[32,35,36]$. The diameter of the visual field is approximately $1.2 \mathrm{~cm}$ in saline. Heparinized saline solution (10 $\mathrm{IU} / \mathrm{mL})$ is then infused, usually at a rate of $5-10 \mathrm{~mL} / \mathrm{s}$, using a power injector to displace the blood between the luminal surface and the fiberscope. The guiding balloon catheter is reshaped for easy placement on the targeted wall segment, usually an "L" or " $U$ " configuration. By slowly pulling back the balloon catheter during the saline infusion, significantly long segments of the aortic luminal surface can be successively visualized (Figure 2(d)). The total amount of saline solution infused should not exceed $500 \mathrm{~mL}$ to avoid acute heart failure due to volume overload.

\subsection{Caval Veins}

Following venography and IVUS, the balloon catheter is introduced in retrograde fashion, and the examination is performed similarly to an aortic examination.

\subsection{Measurement of Lesion Sizes}

Measurement of lesion sizes is beyond the scope of the present AS system because it uses a fish-eye lens. Nevertheless, lesion sizes can be roughly assessed using the diameter of a guide wire tip placed on or adjacent to a lesion.

\section{Combined Use of AS and IVUS}

\section{IVUS System}

In our laboratories, AS is preceded by IVUS because the latter enables successive observation of the entire vascular wall whereas AS is limited to spot observation.

We have developed two IVUS systems; a 5F, $20 \mathrm{MHz}$, 20 cps probe for peripheral vessel use and a 9F, 12 or 20 $\mathrm{MHz}, 20 \mathrm{cps}$ probe for great vessel use.

The arterial and venous systems are usually examined by AS in combination with IVUS. For a femoral arterial examination, an IVUS probe is introduced antegradely into the superficial femoral artery, in which atherosclerotic lesions often occur, guided by a 0.014 inch guide wire. The guide wire is advanced first, and then the probe advanced to the target lesion. The vascular wall is then imaged successively by slowly pulling back the probe.

Following aortography, an IVUS probe for large vessel use is advanced through a femoral artery into the left ventricle, using a 0.035 inch guide wire. Use of a radiofocus guide wire (Terumo Company, Tokyo) is recommended because it is very steerable. Slowly pulling back the probe, pineapple-like images from the left ventricle down to the iliac artery can be obtained successively within $5 \mathrm{~min}$.

AS or IVUS images and fluoroscopic images are simultaneously displayed on a television monitor for confirmation of the location of the area under examination. The details of the procedures are described elsewhere [22,36].

\section{AS Images of Peripheral Artery Disease}

The luminal surface of the non-stenotic peripheral artery as delineated using angiography is smooth surfaced and milky white in color, or light yellow due to fatty streakusing AS. Atherosclerotic plaques that stenose or occlude a peripheral artery are classified by surface morphology into regular (non-ruptured) and complex (ruptured), and by color into white and yellow, as for coronary plaques (Figure 3). Spiral folds are often observed in apparently normal arterieson angiography. Spiral blood streams arethought to induce spiral folds [44].

Thrombus is observed in $20 \%-30 \%$ of cases at the distal end of iliac arterial plaque, and in $30 \%-40 \%$ at the proximal end of the superficial femoral arterial plaque, irrespective of whether regular or complex. Thrombus is usually thin or miniscule and located at the narrowed outlet of the residual lumen in the former, or globular and located around the stenotic inlet in the latter. These thrombi are often not detectable using angiography (Figure 3). It is considered that thrombi are formed by rheological mechanisms, as clearly demonstrated by an experimental study [45].

Distal embolism is not infrequently observed in patients with a trial fibrillation. Typical symptoms are abrupt leg pain and pale decoloration of a lower limb. Figure 4 shows a dark red and globular thrombus occluding the left popliteal artery in a patient with chronic atrial fibrillation. The obstructed segment was successfully recanalized by a Tissue Plasminogen Activator (TPA) infusion followed by balloon angioplasty.

\section{AS and IVUS Images of Aortic Disease}

Atherosclerotic lesions of the aorta are observed in the majority of adult patients, whether symptomatic or asymptomatic.

Atherosclerotic aortic plaques are classified as regular and complex, as for coronary and peripheral arteries [4648]. 

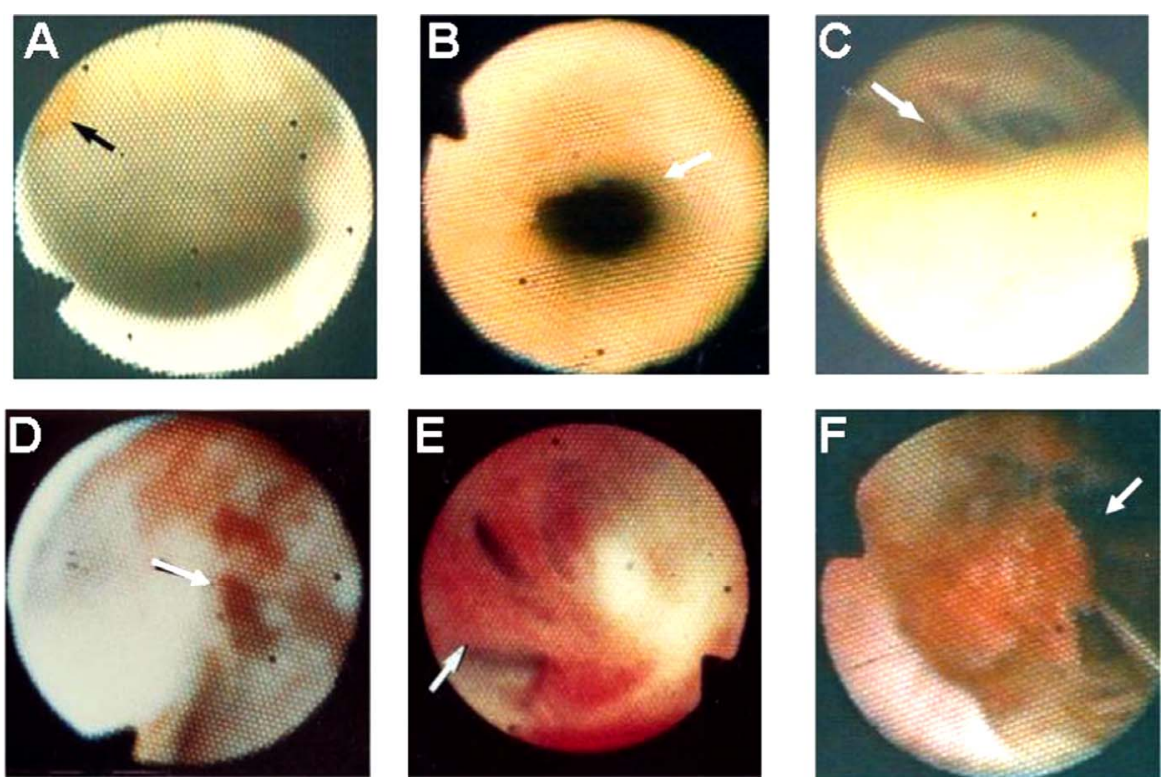

From A to C: non-stenotic left common iliac with a small yellow plaque (arrow), concentric stenosis of a superficial femoral artery with a yellow plaque (arrow), an eccentric and slit-like stenosis of a superficial femoral artery with a yellow plaque (arrow); From D to F: Distal end of a white plaque obstructing the right external iliac artery with tiny red thrombi (arrow), a red thrombus obstructing the distal end of an iliac arterial plaque (arrow), and a red and globular thrombus on the proximal end of a superficial femoral arterial plaque, respectively.

Figure 3. AS images of atherosclerotic plaques and thrombi in peripheral arteries.
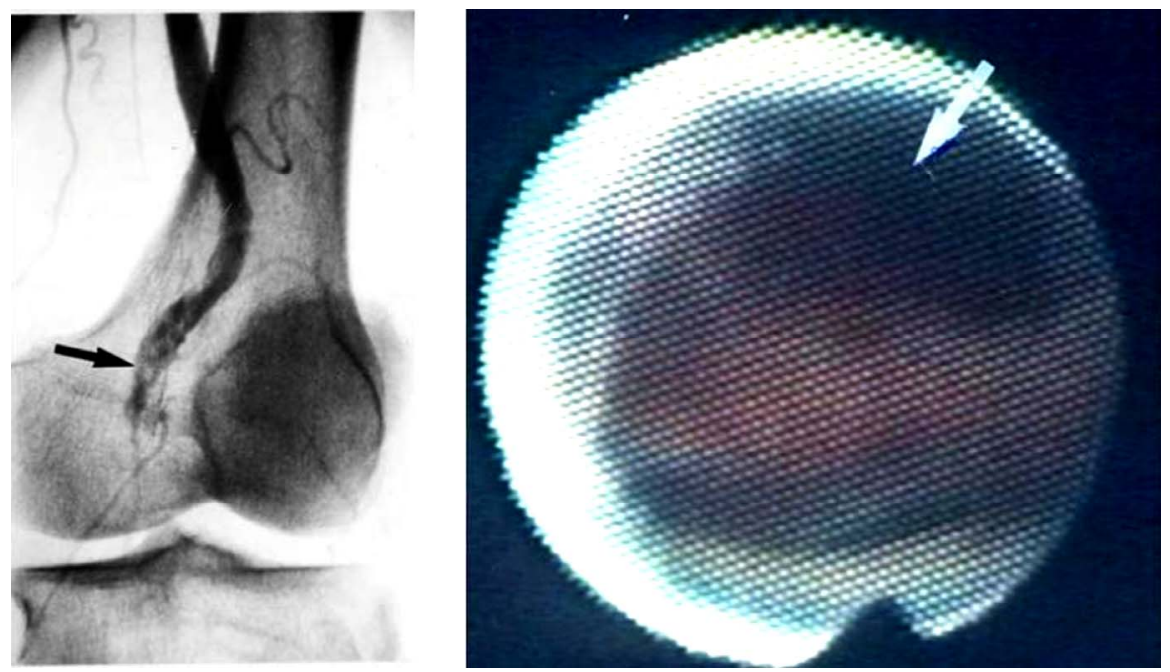

The thromboembolus was dark red, indicating its age is a few days. Cited from reference 48, with permission.

Figure 4. Obstruction of the left popliteal artery with a thromboembolus.

Figure 5 shows representative examples of atherosclerotic aortic changes frequently observed in adult patients. Fatty streaks are observed in the majority of patients. Complex plaques (polypoid or disrupted plaques) are often seen using AS in patients without obvious changes on aortography. Atherosclerotic changes observed by AS are infrequent in the ascending aorta and aortic arch, increase in frequency in the suprarenal abdominal aorta, and are even more common in the intrarenal aorta, where disrupted plaques covered with thrombi are frequently observed (Figure 6). The patients with coronary artery disease have more advanced atherosclerotic changes in the abdominal aorta than those without [22]. Aono observed similar tendency [26].

\subsection{Saccular Aortic Aneurysms}

Saccular (true) aneurysms can appear in any part of the aorta, but occur most frequently in the infrarenal abdominal aorta. 
Figure 7 shows an infrarenal abdominal aorta. Although not detectable using aortography, a large doughnut-like thrombus was detected using IVUS. AS revealed it to be a red thrombus. IVUS is more sensitive in detecting calcification than AS because the latter can only visualize exposed calcifications. In our seriesof 12 patients with abdominal aortic aneurysm, thrombus was ob- served in all, either red, white, or red-and-yellow, indicating thrombi are formed recurrently within aneurysms. Moreover, exposed atheromatous tissue was frequently observed, indicating that plaque disruption was likely induced by distension of the aortic wall [49]. Blood turbulence within aneurysms may also play a part in thrombus formation.
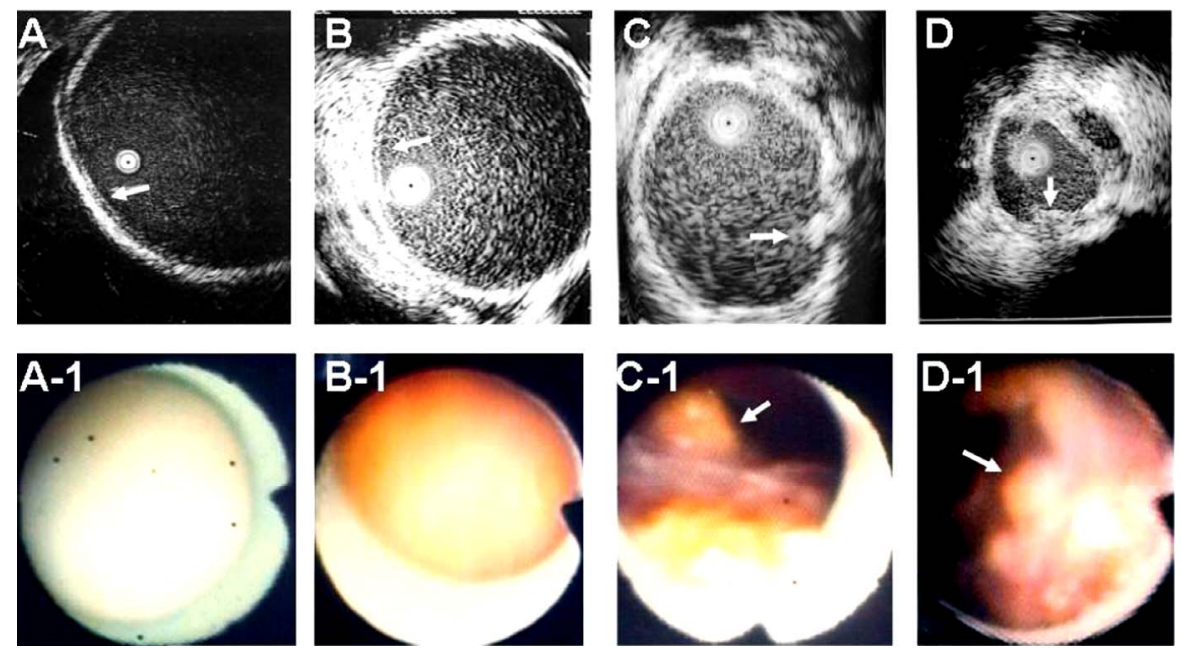

IVUS images of aorta from A to D correspond to the AS images from A-1 to D-1, respectively; A and A-1: The intima was thin ( $<3 \mathrm{~mm}$ in thickness) by IVUS (A) and milky white using AS (A-1), indicating normal aorta. B and B-1: The intima was thick $(>3 \mathrm{~mm})$ using IVUS and yellow using AS, indicating fatty streak. C and C-1: The polypoid plaque using IVUS (arrow) was a yellow polypoid plaque using AS. This plaque belonged to the complex plaque group. D and D-1: A plaque with irregular configuration by IVUS (arrow) was a disrupted plaque using AS (arrow), indicating a complex plaque. Cited from reference 46, with permission.

Figure 5. Aortic lesions observed using IVUS and AS.

A

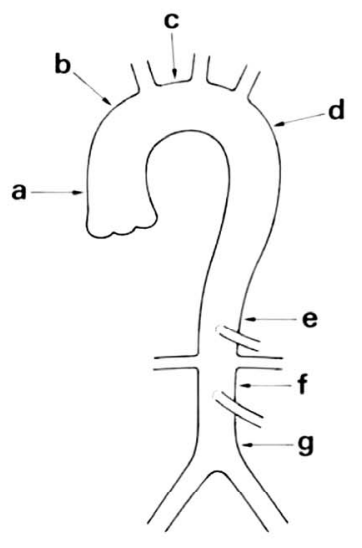

B IVUS

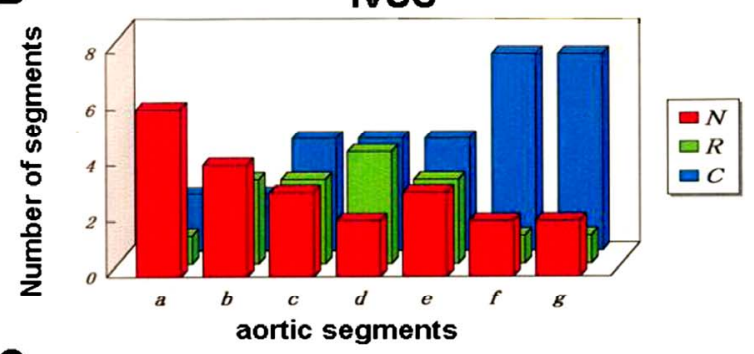

C

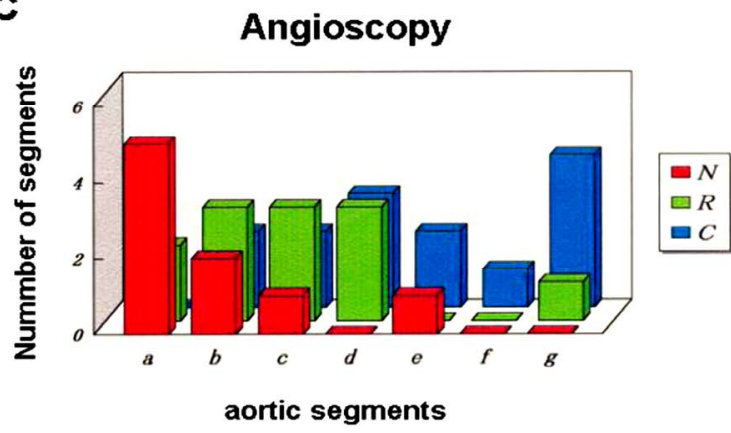

N: No abnormal changes. R: Regular plaque. C: Complex plaque. a to g: Aortic segments observed using AS. Normal segment is frequently seen in the ascending aorta and arch, whereas regular plaques are seen frequently in the descending aorta, and complex plaques are most frequently observed in the abdominal aorta. Cites from reference 46 , with permission.

Figure 6. AS and IVUS changes of the aorta in patients with coronary artery disease. 


\subsection{Dissecting Aneurysm}

Dissecting aneurysms often occur without preceding symptoms. There are no reliable methods for predicting this often fatal condition.
Figure 8 shows AS and IVUS images of an aortic dissection (DeBakey I).

Disrupted intimal flaps at the entry, true and pseudolumens are clearly visualized using IVUS. Since AS is a
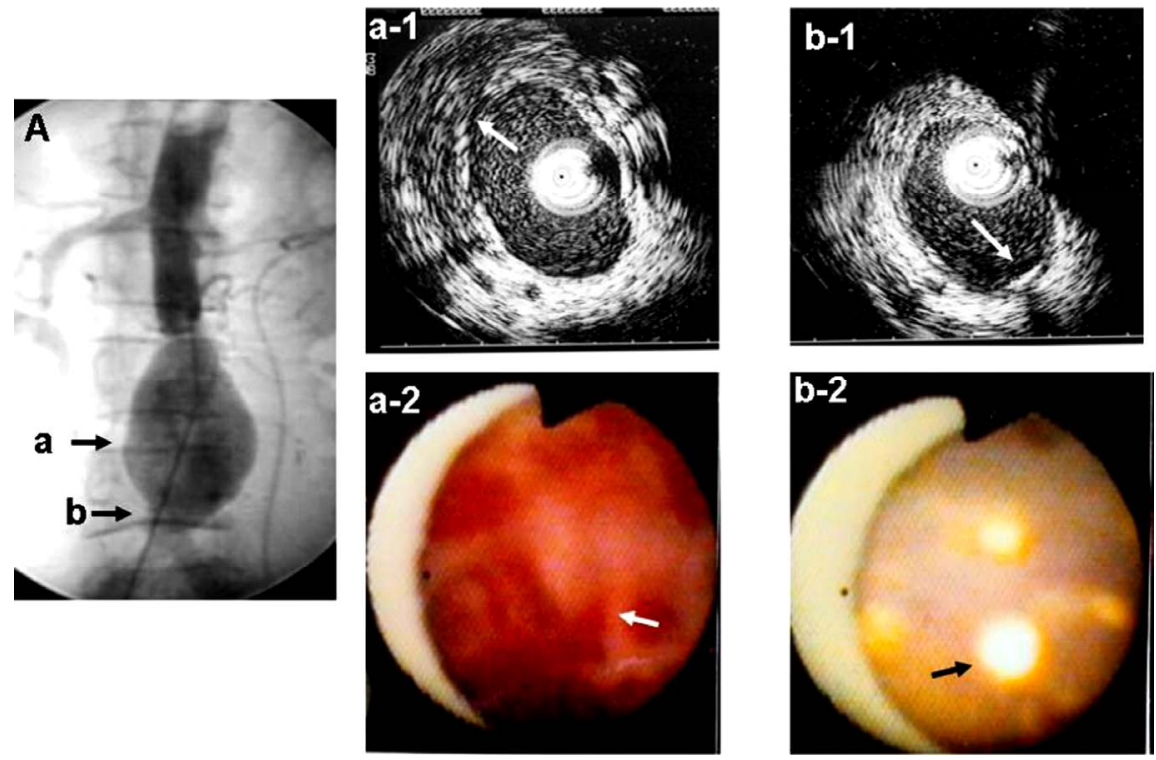

A: (a) and (b) segments examined using both IVUS and AS. A doughnut-shaped thrombus was observed in the aneurysm body using IVUS (a-1). AS revealed it to be a red thrombus (a-2). A calcified plaque was detected in the distal segment of the aneurysm using IVUS (b-1). The calcified plaque reflected light using AS (b-2). Cited from reference 46, with permission.

Figure 7. An infrarenal saccular aneurysm observed in a 72-year-old male.
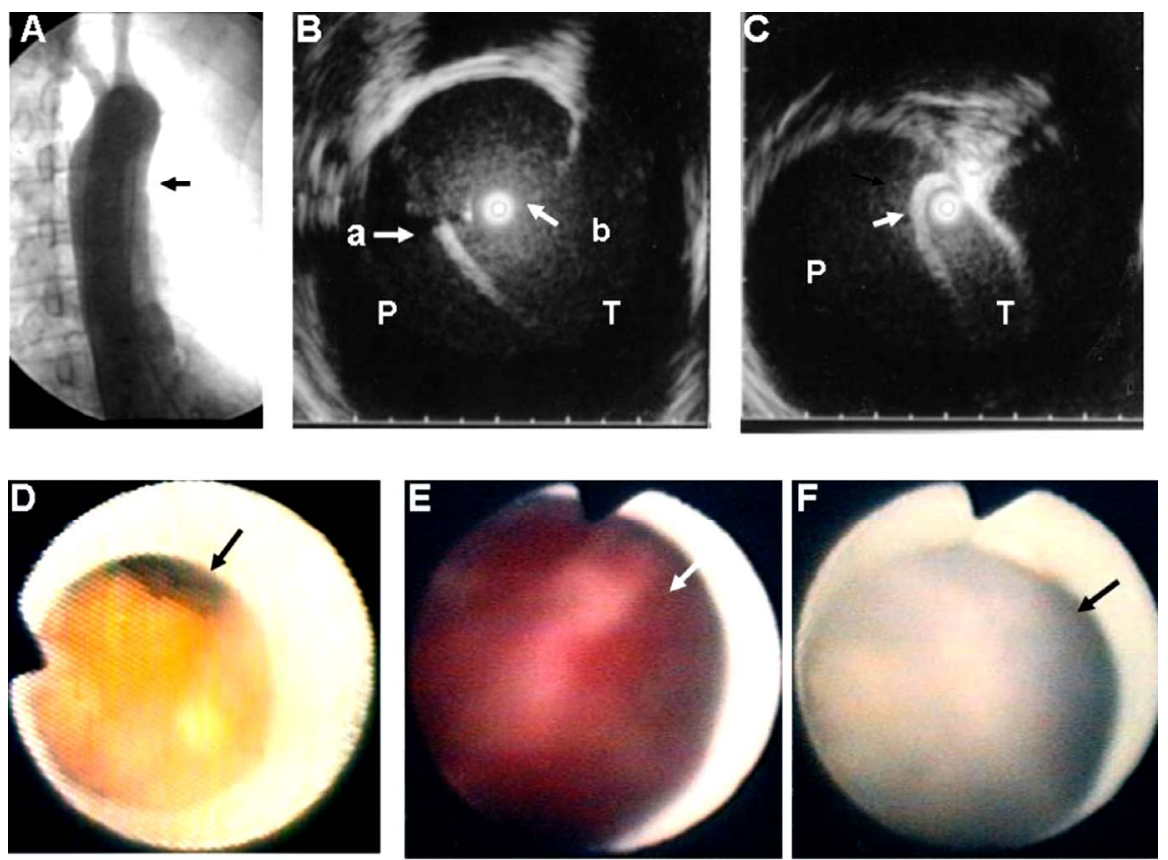

Arrow in an aortogram A: Segment examined using IVUS and AS. B: Intimal flap (a), entry (b), true lumen $(\mathrm{T})$ and pseudolumen (P) confirmed using IVUS. C: Narrowed true lumen in the descending thoracic aorta (T). D: The entry observed using AS was a yellow plaque. E: A mural thrombus in the pseudolumen observed using AS, which accidentally entered into the pseudolumen. F: Intimal flap observed using angioscopy. Blood flow was seen through the pseudolumen. Cited from reference 46, with permission.

Figure 8. Aortic dissection (DeBakey I) observed in a 48-year-old female. 
point-to-point observation, whereas IVUS can survey the entire aortic wall, IVUS is superior to AS in the detection of aortic dissection.

We observed that the entry was surrounded by disrupted yellow matter in the majority of patients, except those with Marfan syndrome or annuloaortic ectasia, suggesting that the yellow plaque is the site of initiation of dissection. It remains to be elucidated what type(s) of yellow plaques are the starting points of dissection.

\section{AS Images of Peripheral Venous Disease}

The major diseases of the peripheral venous system, especially the lower limbs, are varicose veins due to venous valve insufficiency with or without thrombosis, thrombophlebitis, and neoplastic invasion.

Figure 9 shows a normal venous valve leaflet [50]. There are at least three types of valve leaflet abnormality, congenital and acquired, that cause blood regurgitation and consequent varicose veins and leg edema (Figure 10) [27].

Another important venous disease is thrombosis, which not frequently acts as a source of pulmonary emboli. As shown in Figure 11, venous thrombi are classified as mural, often overlooked by phlebography, and globular. Further, they are classified as fresh or organized. When examined using AS, superficial varicous veins often contain fresh or organized thrombi [50].

\section{AS Images of Caval Venous Disease}

Thrombus extending from peripheral veins to the vena cavae is often observed. Also seen are Budd-Chiari disease and tumor thrombus extending from the liver, kidney, or pelvic organs [31]. Figure 11-D-1 shows a tumor thrombus extending from the hepatic vein into the inferior vena cava. Cytology revealedit to be hepatocellular carcinoma.

\section{Evaluation of Intravascular Interventions of Peripheral Arteries Using A}

\subsection{Simultaneous Observation by a Hybrid of AS and IVUS}

We devised a hybrid AS and IVUS system for simultaneous observation of the same target area. This system is composed of a $1.7 \mathrm{~F}$ fiberscope attached to a $5 \mathrm{~F}$ IVUS probe.

\subsection{Balloon Angioplasty}

Figure 12 shows changes in the left superficial femoral artery examined using a hybrid AS/IVUS system. Using this system, the same changes caused by balloon angioplasty could be observed (Figure 12).

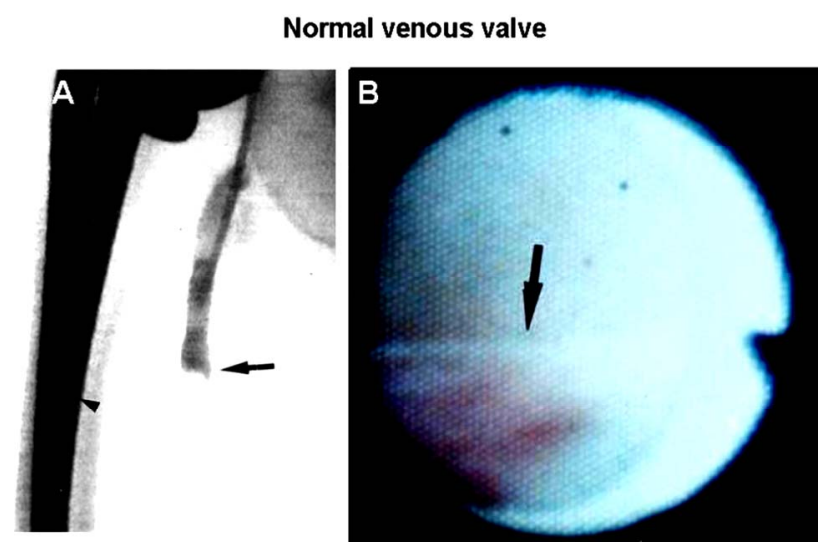

Arrow in A: A venous valve. The venous leaflet was sharp edged. Arrowhead: artificial femur. Cited from reference 50, with permission.

Figure 9. A normal leaflet of a femoral venous valve (arrow).

\section{Anomalous venous valves}

\section{Type I}

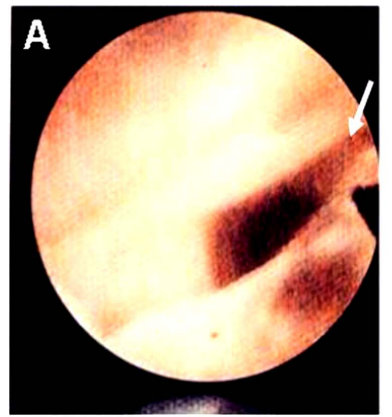

A: Coaptation failure of the two valve leaflets (arrow). B: Partial decay of a leaflet (arrow). C: Perforation in a leaflet (arrow) and coaptation failure (arrowhead). Cited from reference 27, with permission.
Type III
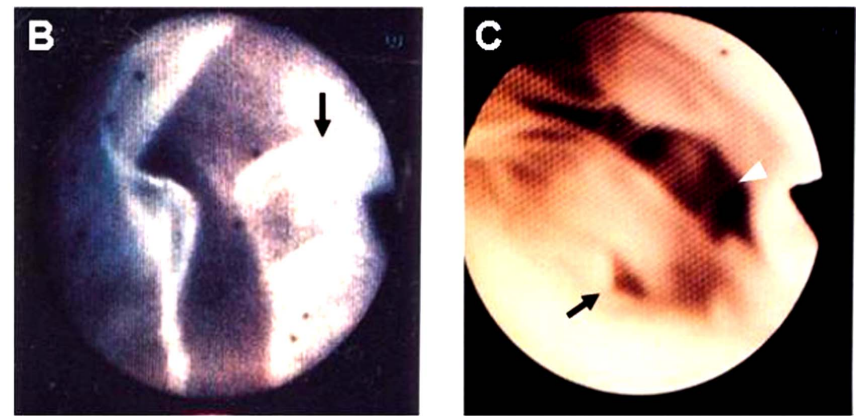

Figure 10. Anomalous venous valves. 

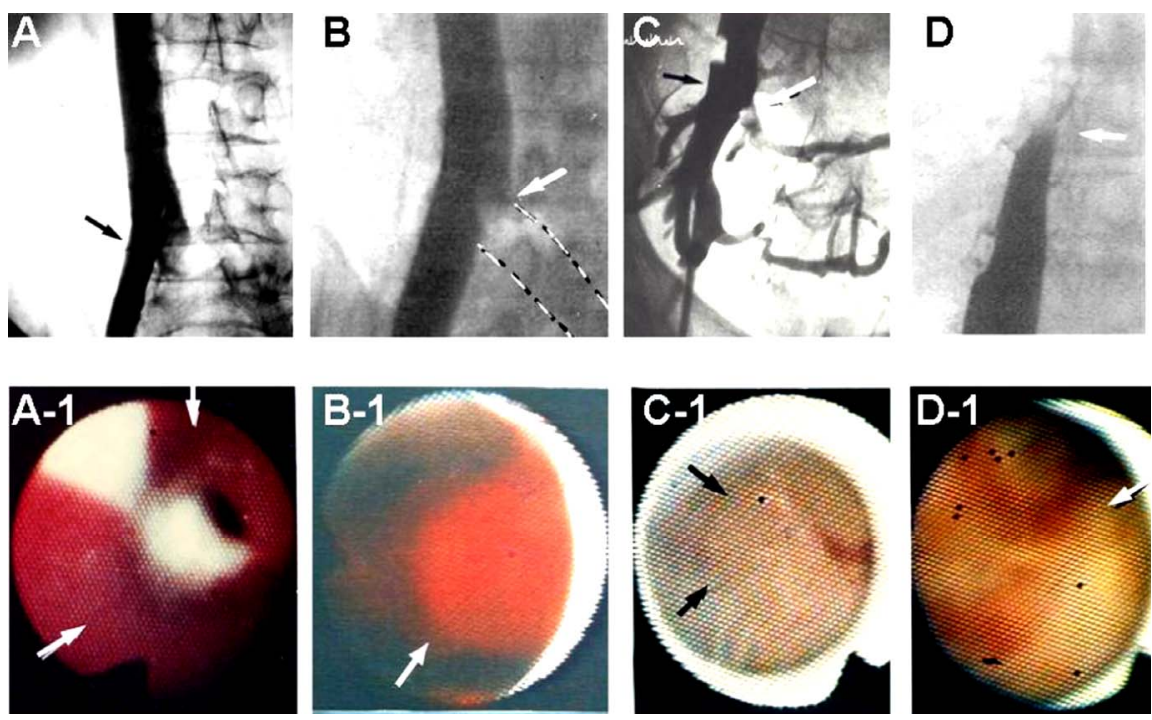

A: Apparently normal right common iliac vein using phlebography. Arrow: segment examined using AS. A-1: A red mural thrombus (arrows) was detected at the site indicated by arrow in A (arrows). B: A defect protruding from left common iliac vein into the inferior vena cava was observed using phlebography (arrow). B-1: AS shows the defect to be a red thrombus protruding from the left common iliac vein (arrow). C: Intrapelvic venous tree. Arrow: Right internal iliac vein. C-1: An organized thrombus was detected in the internal iliac vein (arrow). D: Narrowing of the inferior vena cava (arrow). D-1: The narrowing was revealed to be a tumor thrombus arising from hepatocellular carcinoma and extended through the hepatic vein (arrow). Cited from reference 50 , with permission.

Figure 11. Thrombi in the iliac vein and inferior vena cava.

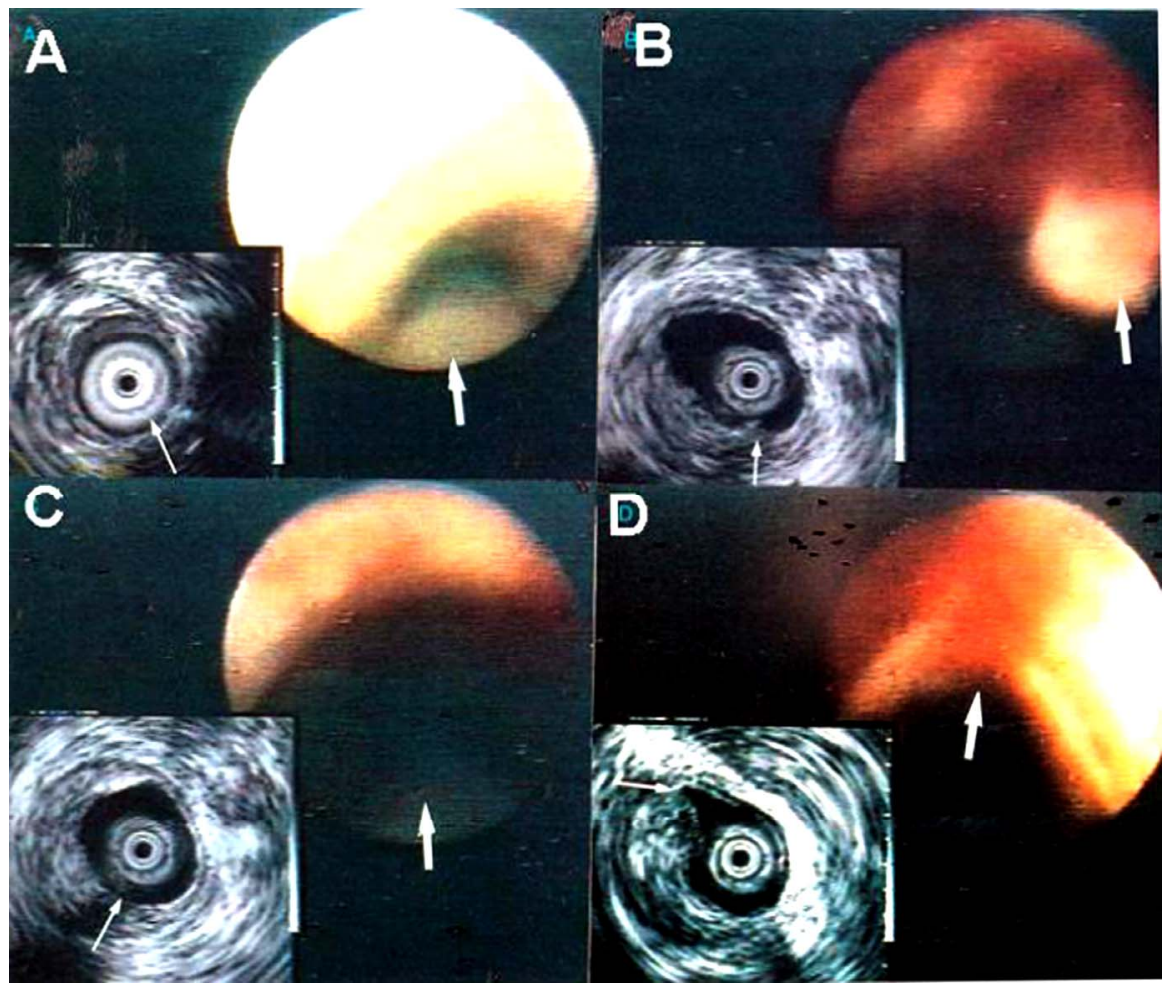

A: Before balloon angioplasty. The stenotic segment was a concentric stenosis using IVUS and a yellow plaque using AS. Arrows: IVUS probe. From B to D: After balloon angioplasty. Intimal flaps in the dilated segment (arrows in B), exposed atheromatous lesions (arrows in C) and a cleft (arrows in D) were observed. Cited from reference 48 , with permission.

Figure 12. Simultaneous evaluation of stenotic lesions of the left superficial femoral artery using a hybrid of IVUS and AS before and after balloon angioplasty. 
Figure 13 shows examples of unwanted changes caused by balloon angioplasty of the iliofemoral arteries, i.e., acute occlusion with intimal flaps, atheromatous tissues and thrombi that often require stenting.

AS is very useful for the immediate diagnosis of the nature of acute occlusion.
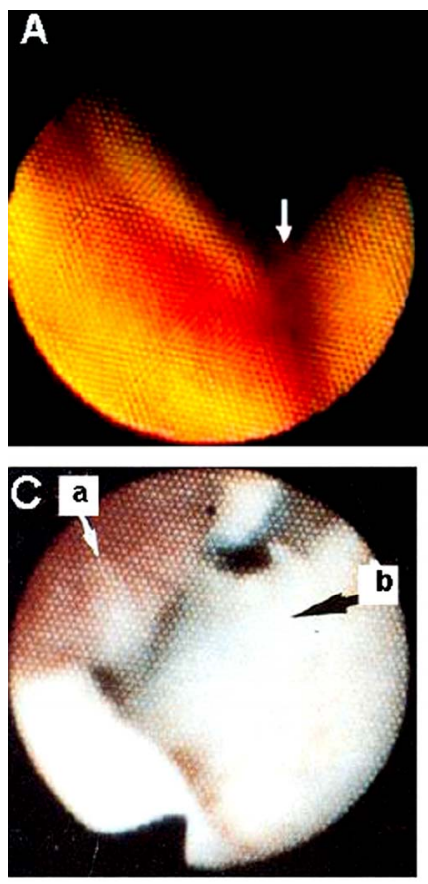

From A to D: A large cleft (arrow in A), occlusion of the dilated lumen with atheromatous tissue (arrow in B), intimal flaps (black arrow in C) and thrombus (white arrow in C) occluding the lumen, and a pseudolumen (arrow) caused by dissection between the intima (white arrowhead) and media (black arrowhead), respectively. Cited from reference 46, with permission.

Figure 13. Various changes in the superficial femoral artery induced by balloon angioplasty.

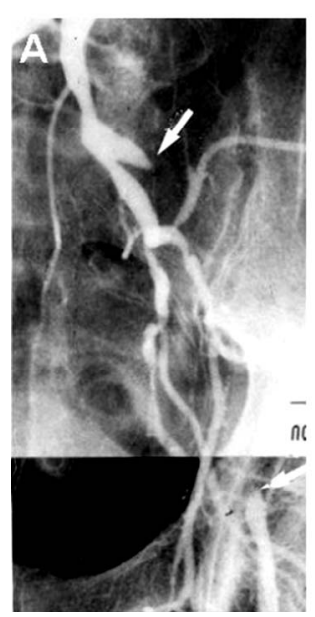

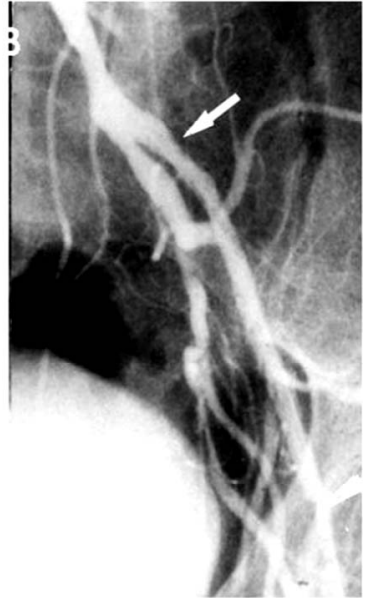

(A)
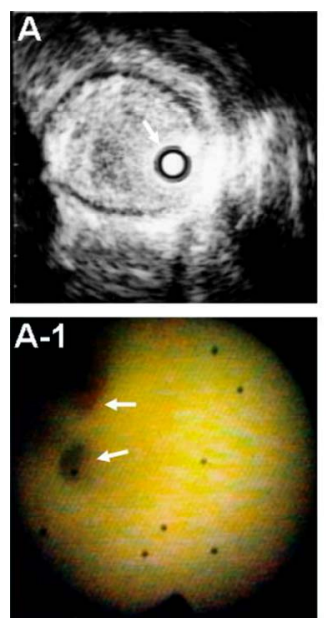
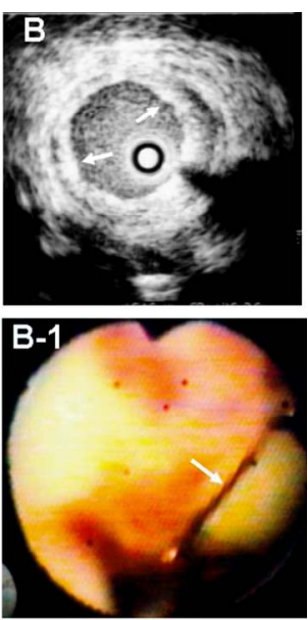

(B)

A: Before stenting. Arrows: occlusion at the site of the proximal arrow; B: recanalization of the occluded segment by stenting; Before stenting, severe narrowing was observed using IVUS (A), with two narrow residual lumens in the distal end of the occluded segment using AS (arrows in A-1). After stenting, recanalization of the segment was confirmed using IVUS (arrows in B), and expanded stent struts using AS (arrow in B-1). Cited from references 52 and 53, with permission.

Figure 14. (A) Total occlusion of the left common iliac artery recanalized by stenting in a 70 year old male; (B) IVUS and AS images before and after stenting in the same patient as in Figure 15(A). 
strut expansion were clearly evaluated by IVUS and AS, respectively.

\subsection{Directional Atherectomy}

Directional atherectomy is used not only for coronary arteries, but also peripheral arteries. Figure 15 shows successful dilatation of the orifice of the left superficial femoral artery using directional atherectomy. The obtained lumen size and cut segment of the plaque were evaluated using IVUS and AS, respectively.

\subsection{Interventional Therapy of Caval Veins}

Stenotic segment of inferior vena cava caused by BuddChiari syndrome or tumor thrombus is also dilated by balloon angioplasty (Figure 16) or stenting.

\section{Evaluation of Surgical Therapies Using AS and IVUS}

\subsection{Open Repair of Saccular Aortic Aneurysm}

The combination of AS and IVUS is very useful in the evaluation of surgical repair of aortic aneurysms.

Figure 17 shows changes in the Y graft one month after open repair. Pseudoaneurysm was found at the sutured segment of the native aortic stump and the graft using both AS and IVUS. Sutures on atheromatous tissues were also found. It is possible that because fragile atheromatous tissues were ligated, the sutures were loosened and

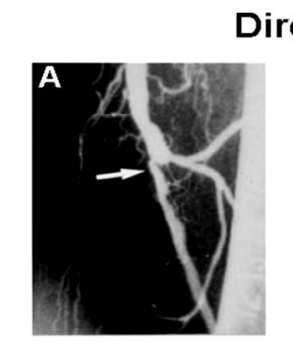

Directional atherectomy
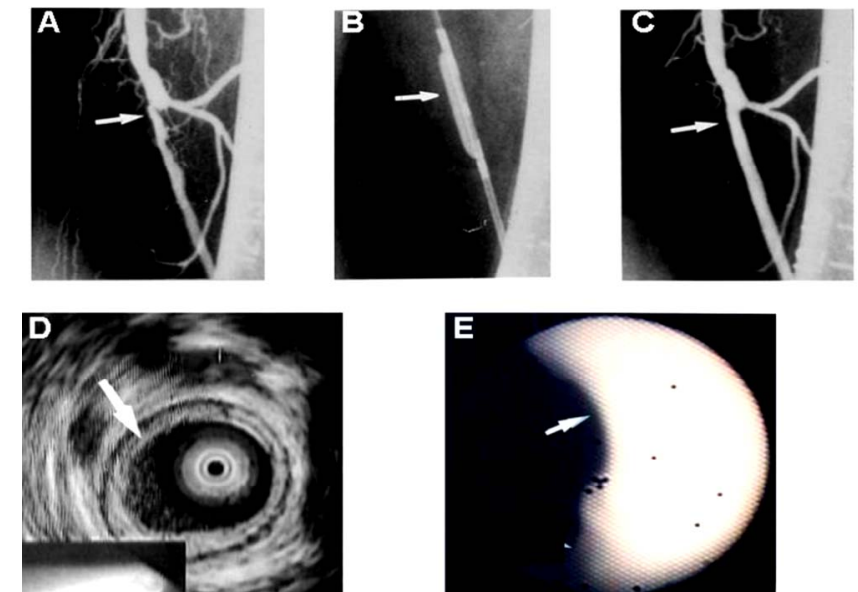

The stenotic SFA (arrow in A) was dilated using atherectomy (B and C). Dilatation was also confirmed using IVUS (D) and AS (E). Cited from reference 52, with permission.

Figure 15. Directional atherectomy of the left superficial femoral artery (SFA) in a 66-year-old male.
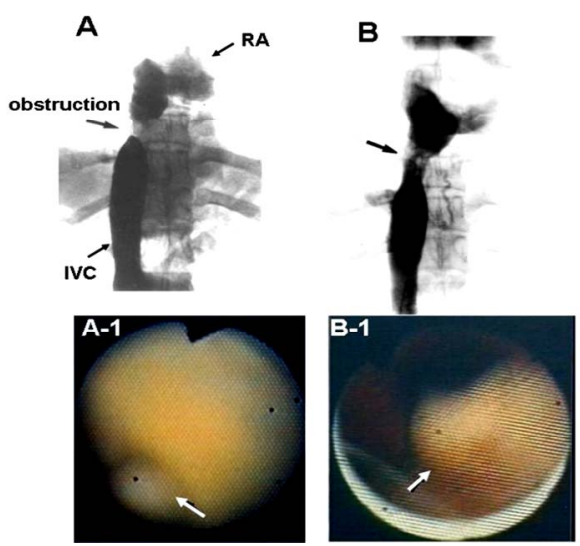

C

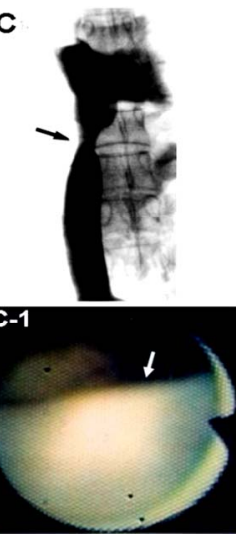

The stenotic proximal segment of the inferior vena cava observed using phlebography (arrow in A) was composed of yellow matter using AS (arrow in A-1). The segment was dilated by balloon angioplasty (B) and clefts were observed using AS (arrow in B). Six months later, restenosis was not observed using phlebography (arrow in C), and the luminal surface of the dilated segment was smooth using AS (arrow in C-1). RA: right atrium; IVC: inferior vena cava. Cited from reference 31 , with permission.

Figure 16. Budd-Chiari syndrome in a 46-year-old female. 
endoleakage occurred, leading to psudoaneurysm formation.

Stentgrafts are now widely used in the treatment of aortic aneurysms. However, migration of the stentgraft and endoleakage occur not infrequently, suggesting the need for precise calibration of the aneurysm neck, and confirmation of the absence of thrombus and fragile atheromatous tissues using AS and IVUS in combination.

\subsection{Open Repair of Annuloaortic Ectasia and Aortic Dissection}

AS and IVUS are now widely used in the examination of pathomorphological changes of the ectatic aortic root in patients with annuloaortic ectasia, solitary or associated with Marfan syndrome.

AS is now being used to predict aortic dissection in this category of aortic diseases, since our recent finding that fold formation in the ectatic aortic root is a morphological change predictive of dissection, irrespective of the luminal diameter [51].

Figure 18 shows changes in the luminal surface of a graft 6 months after repair in a patient with aortic dissection. The luminal surface of the graft is often covered with mural thrombus.

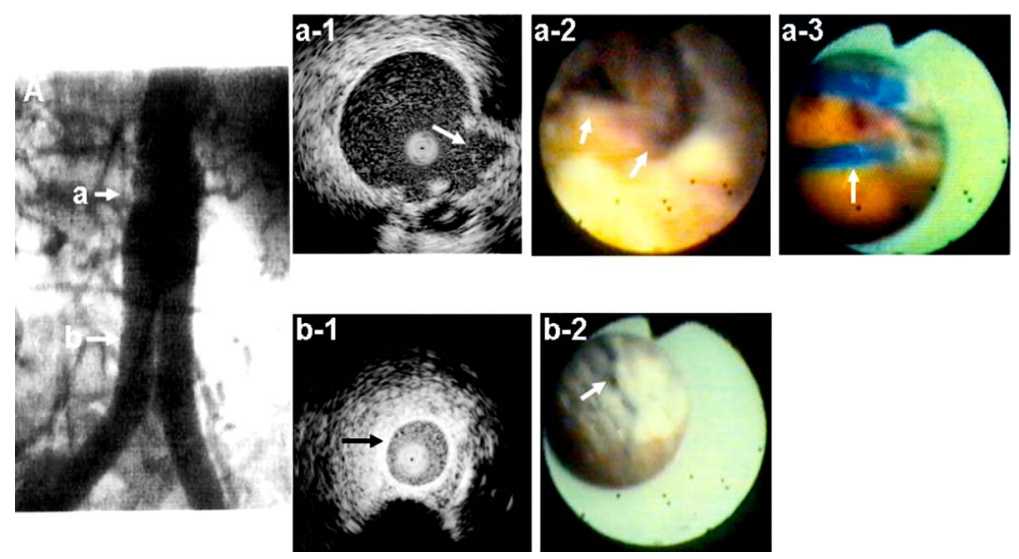

A: Angiogram of the $Y$ graft. a and $b$ : body and right limb of the graft examined using IVUS and AS. A pseudoaneurysm at the sutured site between the native aortic stump and the graft body detected using IVUS (arrow in a-1) and AS (arrows in a-2). Exposed threads were also observed using AS (arrow in a-3). No obstruction was observed in the right limb using IVUS (arrow in b-1), but neointimal coverage of the graft limb was incomplete using AS (arrow inb-2). Cited from reference 49 , with permission.

Figure 17. A 68-year-old male with an infrarenal saccular aneurysm who underwent open repair with a Y graft.
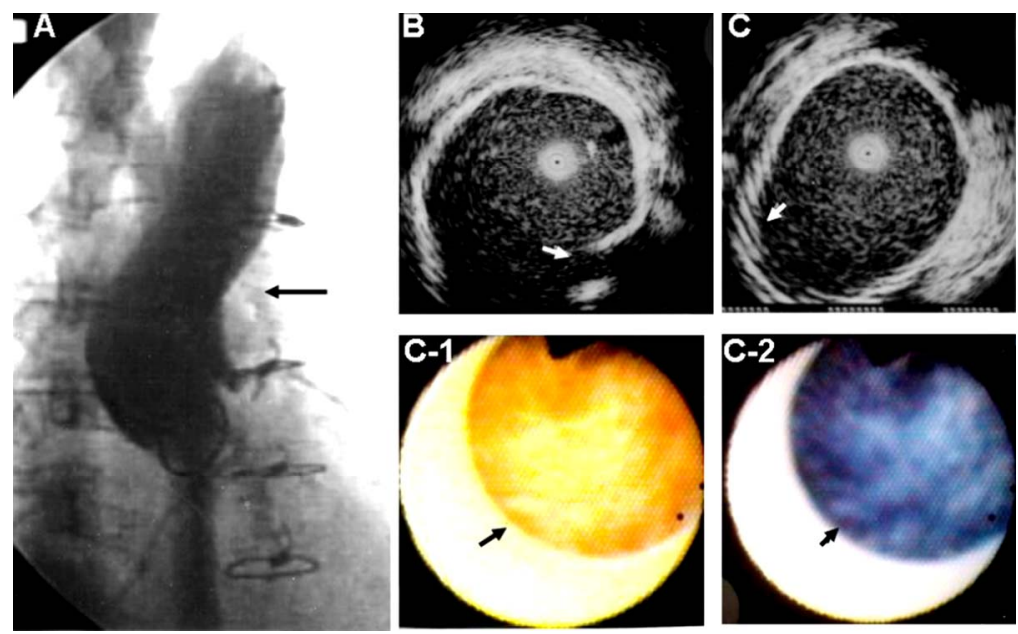

A : Aortogram after grafting (arrow). B: IVUS image of the proximal end of the graft located in the ascending aorta (arrow). C: IVUS image of the graft in the arch. Arrow: bellows of the graft. $\mathrm{C}-1$ : Angioscopic image of the graft which corresponds to C. Red and yellow portions suggest fresh and organized thrombus, respectively (arrow). C-2: Dye staining angioscopic image after topical administration of Evans blue dye. Blue portions indicates fibrin-rich thrombus (arrow). Cited from reference 49 , with permission.

Figure 18. Six months after open repair of aortic dissection with artificial graft. The same patient as that in Figure 8. 


\section{AS-Guided Intravascular Interventions}

\subsection{AS-Guided Intravascular Interventions}

In severely stenosed arteries, the residual lumen is very narrow, often making it difficult to introduce a guide wire into this lumen under fluoroscopic guidance. Angioscopy is a very helpful method for accurate guide wire introduction (Figure 19).

\subsection{AS-Guided Atherectomy}

We developed an AS-guided atherectome to safely remove residual plaquefollowing interventions. A $0.5 \mathrm{~mm}$ fiberscope is attached to an atherectome, which resembles an endomyocardial bioptome (Figure 20(A)). This system is introduced through a 9F guiding balloon catheter into a superficial femoral artery, or iliac artery, to remove residual atheromatous masses (Figure 21) [52].

\section{Angioscope-guided guidewire introduction}
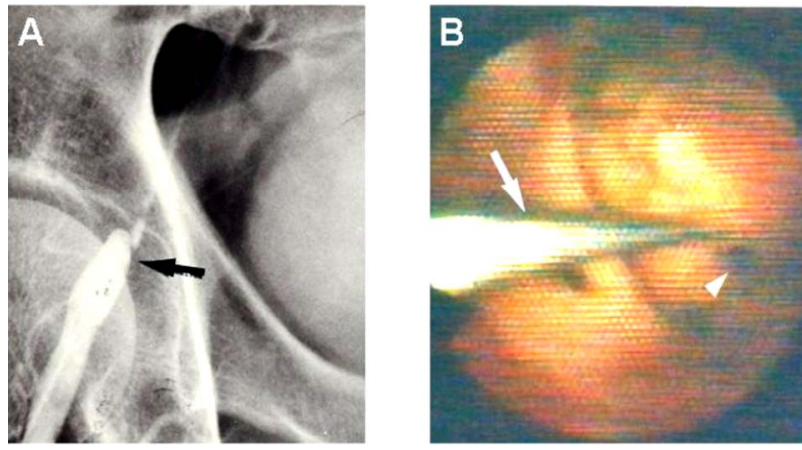

Severely stenosed right external iliac artery with a narrow residual lumen (arrow in A). It was difficult to pass a guidewire through the residual lumen under fluoroscopic guidance, but the guide wire (arrow) could be passed through the lumen (arrowhead) under AS guidance.

Figure 19. AS-guided introduction of a guide wire.

\subsection{Angioscopy-Guided Laser Angioplasty}

In 1990, we devised an AS-guided laser angioplasty system, in which a $1.7 \mathrm{~F}$ fiberscope is fixed onto a laser probe for temperature-controlled thermal angioplasty using an Nd-YAG laser (Figure 20(B)). Figure 22 shows a representative patient in whom a long segment occlusion of the right superficial femoral artery was successfully recanalized using this system, and the process of recanalization could be monitored successively with AS [53].

\subsection{AS-Guided Venous Valvuloplasty}

Varicose veins of the legs are caused by acquired or congenital venous valve abnormalities. This is a troublesome disease, not only from the medical standpoint, but also the cosmetic aspect, especially for women. ASguided valvuloplasty has been intensively performed by

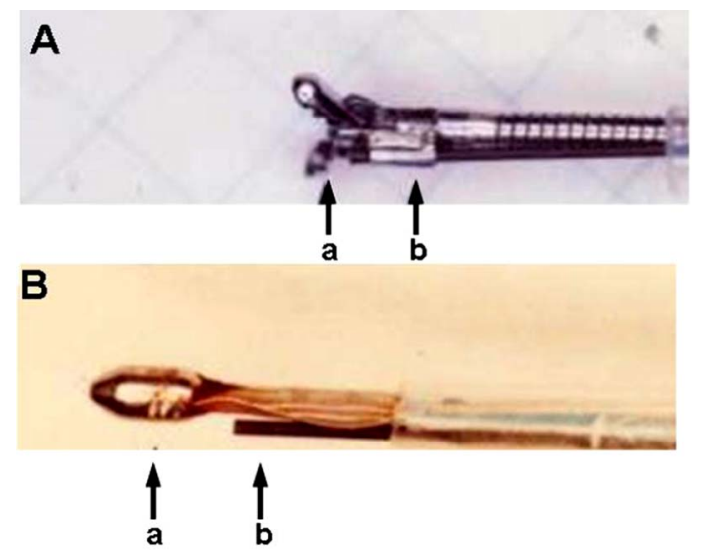

A: Angioscope-guided atherectome. a: atherectome. b: fiberscope; B: Angioscope-guided laser probe. a: laser probe. b: fiberscope; Cited from reference 52, with permission.

Figure 20. AS-guided therapeutic tools.

\section{Angioscope-guided atherectomy}
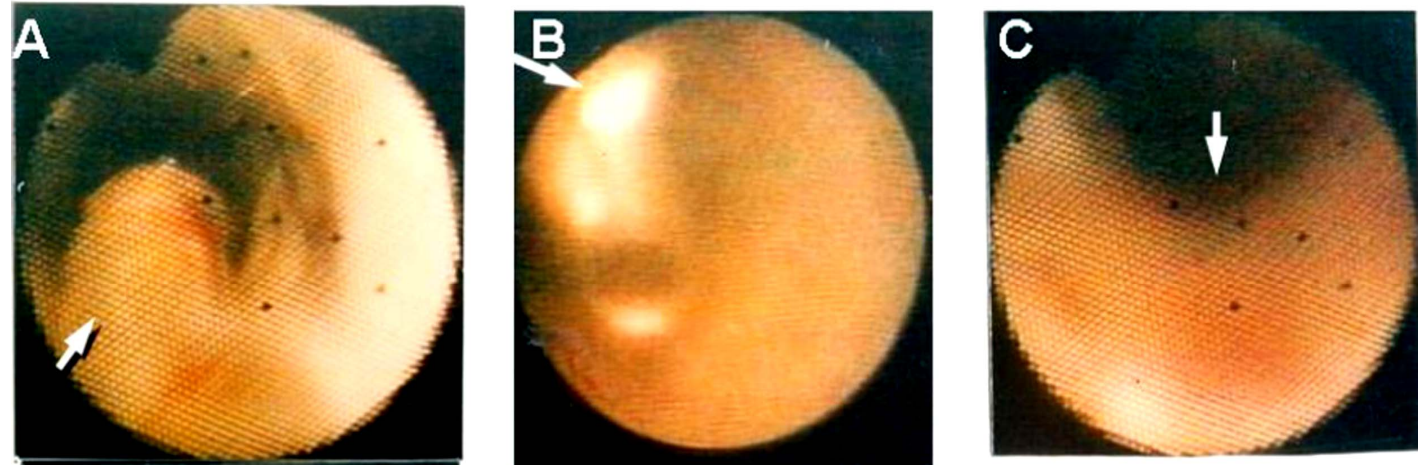

A: Atheroma protruding into the lumen following balloon angioplasty (arrow); B: During removal of the atheroma by the atherectome Arrow: atherectome tip; C: Removal of the atheromatous tissue by atherectomy was confirmed using AS (arrow).

Figure 21. Removal of residual atheroma by an AS-guided atherectome. 


\section{Angioscope-guided laser angioplasty}
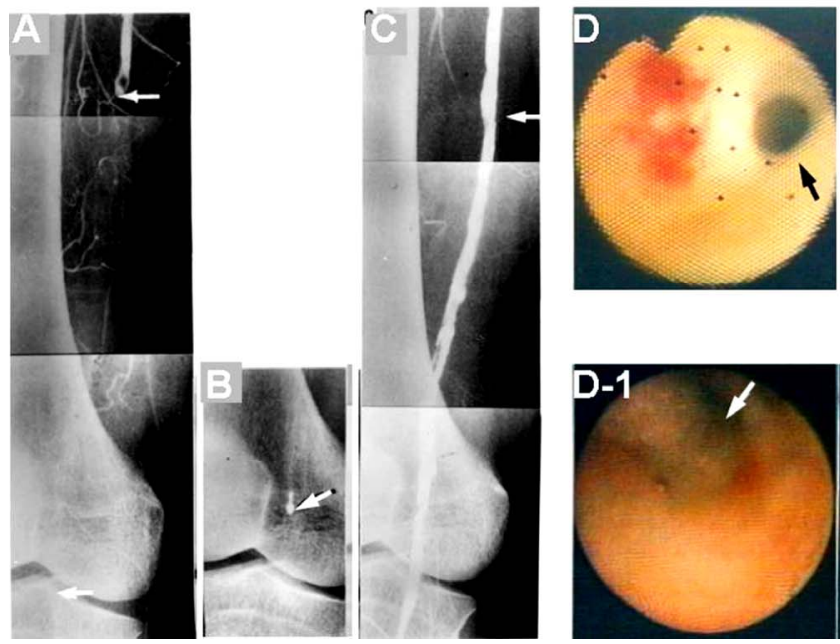

A: Total occlusion of the right superficial femoral artery observed using angiography (arrows); B: During thermal laser angioplasty. Arrow: laser tip; C: Complete recanalization after laser angioplasty (arrows), confirmed using angiography; D: Angioscopic appearance before laser angioplasty. Using AS, the proximal end of the occluded segment was composed of a yellow plaque with a small red thrombus on it. Arrow: small residual lumen; D-1: During laser angioplasty. The laser probe can be clearly seen using AS (arrow); D-2: After laser angioplasty. The occluded segment was recanalized, but carbonized tissue remained on the luminal surface (arrow). Cited from reference 53, with permission.

Figure 22. A 70-year-old male with total occlusion of the right superficial femoral artery who underwent AS-guided thermal laser angioplasty.

\section{Venous valvuloplasty}

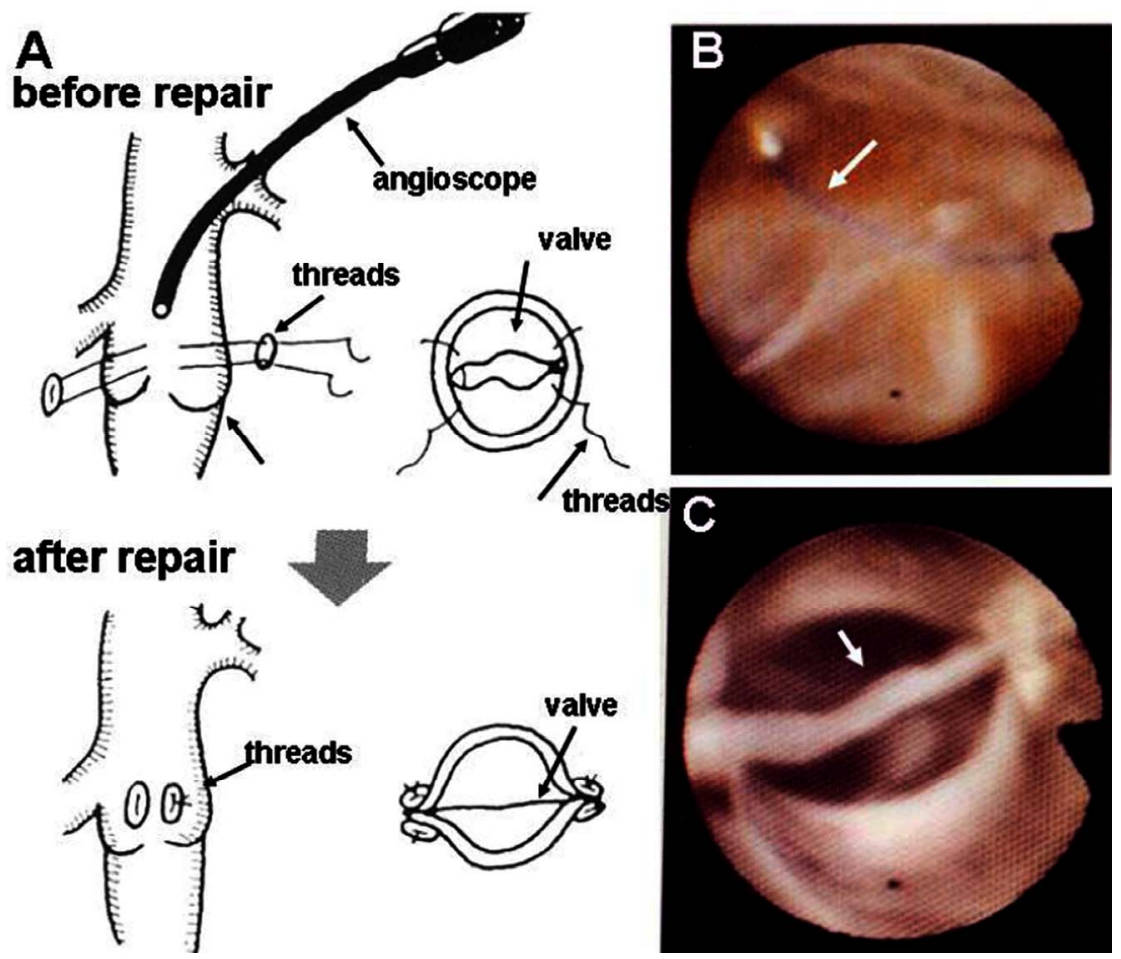

A: Schematic representation of venous valvuloplasty procedure; B: During valvuloplasty. Arrow: suture needle; C: Following valvuloplasty. Complete coaptation of the two valve leaflets was achieved (arrow). Cited from reference 25, with permission.

Figure 23. AS-guided valvuloplasty of femoral vein. 
Hoshino and his coworkers, with successful results (Figure 23) [27]. Recently, AS-guided venoplasty of the saphenous vein has been widely performed, with beneficial effects.

\section{New AS Techniques}

\subsection{Dye-Staining AS for Tissue Imaging}

In the field of coronary AS, dye-staining AS is widely used for molecular or tissue imaging of the coronary wall and thrombi using biocompatible and low-molecular dyes. This technology is not yet used systematically in any areas other than coronary disease [39].

Figure 24 shows white-to-yellow matter in the abdominal aorta. This matter exhibited blue and white colors in a mosaic pattern after topical application of Evans blue dye, which stains fibrin blue but not stain platelet aggre- gates, thereby indicating that the matter was composed of fibrin dominant segments and platelet dominant segments. Following additional application of fluorescein, which stains platelets, the white segments exhibited fluorescence, by excitation at $470 \mathrm{~nm}$ and emission at 515 $\mathrm{nm}$, confirming that the white segments were composed of platelets.

\subsection{Cellular Imaging Using Intravascular Microscopy}

We devised a rigid angiomicroscope that magnifies the target up to $\times 350$. Use of this angiomicroscope is limited to straight vessels such as the femoral or iliac artery, not curved or tortuous vessels (Figure 25).

Using this angiomicroscope, foam cells in the disrupted plaque can be clearly discerned (Figure 26) [38].

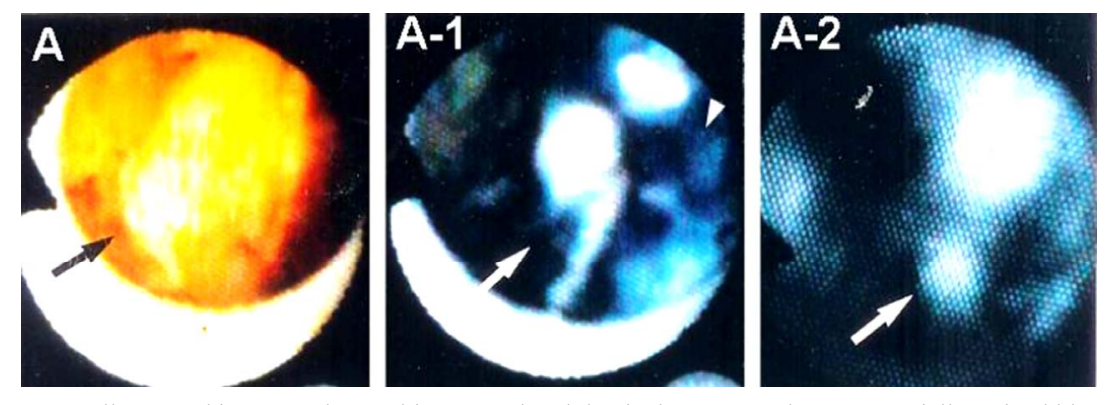

A: A yellow-to-white mass observed in a saccular abdominal aorta; B: The mass partially stained blue with Evans blue dye but other segments remained white (arrow); C: The white segment of the mass exhibited fluorescence after administration of fluorescein, indicating that this segment was composed of platelets.

Figure 24. Dye-staining and fluorescent AS of thrombus composition.
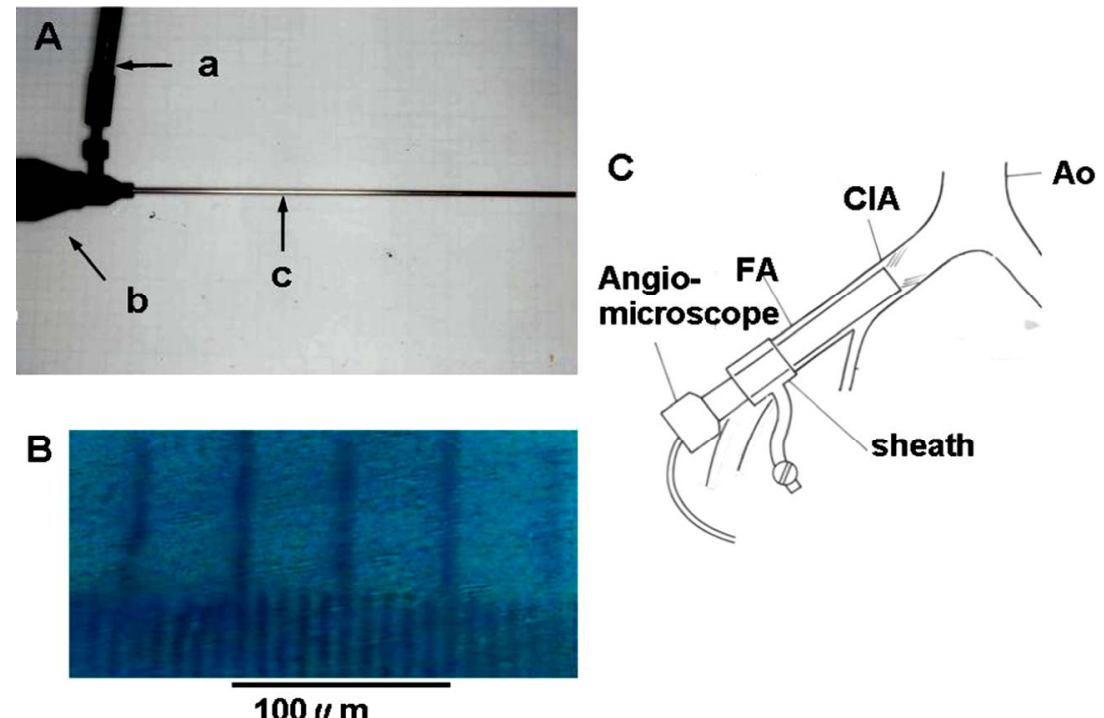

$100 \mu \mathrm{m}$

A: Lateral view of the angiomicroscope. a: light guide. b: image guide. c: rod lens; B: Scale observed using the angiomicroscope. The discrimination power was $10 \mu \mathrm{m}$; $\mathrm{C}$ : Schematic representation of angiomicroscopic procedure. FA: femoral artery; CIA: common iliac artery; Ao: aorta. Cited from references 34 and 35 , with permission.

Figure 25. Angiomicroscope for cellular imaging. 

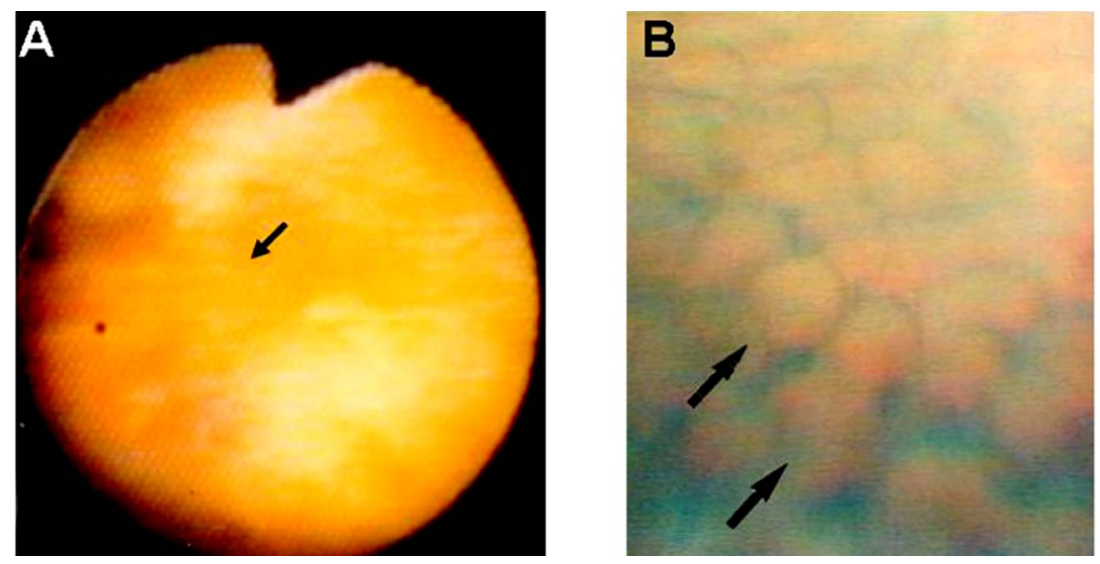

A: The luminal surface of the dilated iliac segment examined using a conventional angioscope. Arrow: the segment observed using angiomicroscopy; B: Foam cells observed using angiomicroscopy (arrow); Cited from reference 35 , with permission.

Figure 26. The right external iliac artery examined using an angiomicroscope following balloon angioplasty in a 62-year-old male.

\subsection{Molecular Imaging Using Fluorescent AS}

Molecular imaging of substances comprising atherosclerotic plaques has been intensively studied using a variety of imaging techniques. We succeeded in in vivo imaging of oxidized low density lipoprotein in the coronary artery wall of patients with coronary artery disease $[40,41,54]$. This technique may contribute to the understanding and evaluation of molecular targeted therapies of vascular disease.

\section{Future Prospects}

The major cause of aortic and peripheral artery diseases is atherosclerosis, as in the case of coronary artery disease. Until now, a number of imaging techniques have been applied to the imaging of the cells such as macrophages, and substances comprising atherosclerotic plaques such as lipids (cholesterol, cholesterol esters and triglyceride), lipoproteins, apolipoproteins, calcium compounds and enzymes, in order to clarify the molecular mechanisms of atherosclerotic disease, as well as vascritis such as arteritis and aortitis, the mechanism of which are not well understood.

Although invasive, angioscopy is a high resolution imaging technique and therefore useful for molecular imaging and for the guidance and evaluation of molecular targeted therapy for vascular disease. Clinical application of fluorescent angioscopy has commenced for molecular imaging of coronary and myocardial disease. This technique will soon be applied to peripheral and great vessels in the clinical situation.

\section{Conclusions}

Recent advances in AS technology allow us to examine the vascular interior. This imaging technology is now used for the diagnosis of vascular diseases, evaluation of surgical and interventional therapies, and guidance of interventions. AS will also be employed, as in coronary artery disease, for cellular and molecular imaging to clarify the underlying mechanisms of congenital and acquired vascular diseases, and for molecular targeted therapy.

We developed or obtained the AS systems and data presented in this article prior to 2000 . We anticipate that new imaging technologies will be developed and further contribute to the diagnosis and treatment of vascular disease.

\section{REFERENCES}

[1] D. S. Allen and E. A. Graham, "Intracardiac Surgery-A New Method," The Journal of the American Medical Association, Vol. 79, No. 13, 1922, pp. 1028-1030.

[2] D. E. Harken and E. M. Glidden, "Experiments in Intracardiac Surgery. II. Intracardiac Visualization," The Journal of Thoracic and Cardiovascular Surgery, Vol. 12, No. 4, 1943, pp. 566-573.

[3] H. Sakakibara, T. Ichikawa and J. Hattori, "An Intraoperative Method for Observation of Cardiac Septal Defect Using a Cardio Scope," Operation, Vol. 10, 1956, pp. 285290.

[4] H. Sakakibara, T. Iijima and J. Hattori, "Direct Visual Operation for Aortic Stenosis: Cardioscope Studies," The Journal of the International College of Surgeons, Vol. 29, No. 5, 1958, pp. 548-552.

[5] S. Sugie and T. Tanabe, "Observation of the Vascular Movements by Vascular Endoscope," Naika, Vol. 24, No. 2, 1969, pp. 277-279.

[6] F. Litvack, W. S. Grundfest, M. E. Lee, R. M. Carrol, R. Fran, A. Chaux, G. Berci, H. B. Rose, J. M. Matroff and J. S. Forrester, "Angioscopic Visualization of Blood Vessel Interior in Animals and Humans," Clinical Cardiology, Vol. 8, No. 2, 1985, pp. 65-70. 
doi:10.1002/clc.4960080202

[7] Y. Uchida, T. Tomru, F. Nakamura, H. Sonoki and T. Sugimoto, "Fiberoptic Angioscopy of Cardiac Chambers, Valves and Great Vessels Using a Guiding Balloon Catheter in Dogs," American Heart Journal, Vol. 1118, No. 6, 1988, pp. 1297-1302. doi:10.1016/0002-8703(88)90024-5

[8] M. J. Buckmaster, G. J. Hyde, T. J. Nypaver, E. D. Endean, T. H. Schwarcz and C. S. Kuo, "An Angioscopic Method for Intraluminal Aortic Evaluation and Stent Placement," Journal of Vascular Surgery, Vol. 21, No. 5, 1995, pp. 818-821. doi:10.1016/S0741-5214(05)80013-8

[9] J. R. Spears, A. M. Spokojny and H. J. Marais, "Coronary Angioscopy during Cardiac Catheterization," Journal of the American College of Cardiology, Vol. 6, No. 1, 1985, pp. 93-97. doi:10.1016/S0735-1097(85)80258-8

[10] Y, Uchida, S. Masuo, T. Tomaru and A. Kato, "Fiberoptic Observation of Coronary Luminal Changes Caused by Transluminal Coronary Angioplasty," Circulation, Vol. 72, No. 3, 1985, p. 219.

[11] T. A. Sanborn, J. A. Rygaard, B. M. Westbrook, H. L. Lazar, G. McCormick, A. J. Roberts and I. Madroff, "Intraoperative Angioscopy of Saphenous Vein and Coronary Arteries," The Thoracic and Cardiovascular Surgeon, Vol. 91, No. 3, 1986, pp. 339-343.

[12] V. Hombach, M. Hoeher, A. Hannekum, W. Hugel, B. Buran, H. W. Hoeppe and H. Hirche, "Erste Klinische Erfarlungen Mit Koronarendoskopie," Deutsch Medi Wochenschr, Vol. 111, No. 30, 1986, pp. 1135-1140. doi:10.1055/s-2008-1068597

[13] C. T. Sherman, F. Litvak, W. Brundfest, M. Lee, A. Hickey, A. Chaux, R. Kass, C. Blanche, J. Matroff, L. Morgenstein, W. Ganz, H. J. C. Swan and J. Forrester, "Coronary Angioscopy in Patients with Unstable Angina Pectoris," The New England Journal of Medicine, Vol. 315, No. 15, 1986, pp. 909-919.

[14] Y. Uchida, K. Hasegawa, K. Kawamura and I. Shibuya, "Angioscopic Observation of the Coronary Luminal Changes Induced by Percutaneous Transluminal Angioplasty," American Heart Journal, Vol. 117, No. 4, 1989, pp. 769-776. doi:10.1016/0002-8703(89)90611-X

[15] F. Nakamura, J. Kvasnicka, Y. Uchida and H. J. Geshwind, "Percutaneous Angioscopic Evaluation of Luminal Changes Induced by Excimer Laser Angioplasty," American Heart Journal, Vol. 124, No. 6, 1992, pp. 1467-1472. doi:10.1016/0002-8703(92)90058-4

[16] Y. Uchida, "Percutaneous Cardiovascular Angioscopy," In: G. S. Abela, Ed., Lasers in Medicine and Surgery, Kluwer Academic Publishers, Boston, 1990, pp. 399-410.

[17] V. Hombach, M. Hoeler, M. Koches, T. Eggeling, A. Schmidt, H. W. Hoep and H. H. Hilger, "Pathophysiology of Unstable Angina Pectoris: Correlations with Coronary Angioscopic Images," European Heart Journal, Vol. 9, 1998, pp. 140-145.

[18] Y. Uchida, Y. Fujimori, T. Tomaru, O. Oshima and J. Hirose, "Percutaneous Angioplasty of Chronic Obstruction of Peripheral Arteries by a Temperature-Controlled NdYAG Laser System," Journal of Interventional Cardiology, Vol. 5, No. 4, 1992, pp. 301-308.

doi:10.1111/j.1540-8183.1992.tb00834.x
[19] G. Robinski, D. Brisset, F. Phillippe, D. Kremer, C. Layrian, G. Montalescot and D. Thomas, "Effects of Ultrasound Energy on Total Peripheral Artery Occlusions: Initial Angiographic and Angioscopic Results," Journal of Interventional Cardiology, Vol. 6, No. 2, 1993, pp. 157163. doi:10.1111/j.1540-8183.1993.tb00848.x

[20] L. Kollar, G. Kasza, G. Rozsos, G. Menyhei, M. Szabo and L. Horvath, "Internal Carotid Stent Implantation with Angioscopic Control," Acta Chirurgica Hungarica, Vol. 36, No. 1-4, 1997, pp. 168-169.

[21] W. Trubel, H. Magometschnigg, Y. al-Hachichi, M. Staudacher, E. Wolner and P. Polterauer, "Intraoperative Control Following Femorodistal Revascularization: Angioscopy Is Superior to Angiography," The Thoracic and Cardiovascular Surgeon, Vol. 42, No. 4, 1999, pp. 199-207. doi:10.1055/s-2007-1016488

[22] Y. Uchida, “Aortoscopy,” In: Y. Uchida, Ed., Atlas of Cardioangioscopy, Medical View Publishing, Tokyo, 1995, pp. 180-188.

[23] B. B. Hill, R. Neville, G. L. Hyde, C. S. Kuo and E. B. Diethrich, "Angioscopic Evaluation of an Endoluminal Aortic Graft: The First Clinical Experience," Journal of Endovascular Surgery, Vol. 2, No. 3, 1995, pp. 248-254. doi:10.1583/1074-6218(1995)002<0248:AEOAEA >2.0.C $\underline{\mathrm{O} ; 2}$

[24] K. Tokuhiro, Y. Uchida, K. Kawamura, H. Sakuragawa, H. Masuhara, H. Oosawa and N. Koyama, "Evaluation of Annuloaortic Ectasia by Angioscopy and IVUS," Diagnostic and Therapeutic Endoscopy, Vol. 7, No. 1, 2000, pp. 35-45. doi:10.1155/DTE.7.35

[25] K. Tsagakis, M. Kamler, J. Benedik and H. Jakob, “Angioscopy-A Valuable Tool in Guiding Hybrid Stent Grafting and Decision Making During Type A Aortic Dissection Surgery," European Journal Cardio-Thoracic Surgery, Vol. 38, No. 4, 2010, pp. 507-509.

doi:10.1016/j.ejcts.2010.02.010

[26] J. Aono, K. Watanabe, H. Shimizu, H. Higashi, K. Oshima, K. Ishibashi, S. Ikeda and M. Hamada, European Heart Journal, Vol. 28, No. 1, 2007, p. 881.

[27] S. Hoshino, H. Sadokawa, F. Iwaya, T. Igari, T. Ono and S. Takase, "Externalvalvuloplasty under Preoperative Angioscopic Control," Phlebologie, Vol. 47, 1993, pp. 521529.

[28] T. Yamaki, K. Sasaki and M. Nozaki, "Preoperative Duplex-Derived Parameters and Angioscopic Evidence of Valvular Incompetence Associated with Superficial Venous Insufficiency," Journal of Endovascular Therapy, Vol. 9, No. 2, 2002, pp. 229-233.

doi:10.1583/1545-1550(2002)009<0229:PDDPAA $>2.0 . \mathrm{C}$ $\mathrm{O} ; 2$

[29] T. Nishibe, F. Kudo, K. Miyazaki, Y. Kondo, J. Koizumi, A. Dardik and M. Nishibe, "Intermediate-Term Results of Angioscopy-Assisted Anterior Valve Sinus Placation for Primary Deep Venous Insufficiency," The Journal of Cardiovascular Surgery, Vol. 48, No. 1, 2007, pp. 21-25.

[30] H. Komai and M. Juri, "Deep Venous External Valvuloplasty Using a Rigid Angioscope," Surgery Today, Vol. 40 , No. 6, 2010, pp. 538-542. doi:10.1007/s00595-009-4076-8 
[31] Y. Uchida, “Angioscopy of Caval Veins," In: Y. Uchida Ed., Atlas of Cardioangioscopy, Medical View Ltd., Tokyo, 1995, pp. 206-216.

[32] D. Shure, G. Grigoratos and K. M. Moser, "Fiberoptic Angioscopy. Role in the Diagnosis of Chronic Pulmonary Arterial Obstructions," American Board of Internal Medicine, Vol. 103, No. 6, 1985, pp. 844-850.

[33] Y. Uchida, T. Oshma and J. Hirose, "Angioscopic Detection of Residual Pulmonary Thrombi in the Differential Diagnosis of Pulmonary Thromboembolism," American Heart Journal, Vol. 130, No. 4, 1995, pp. 854-859. doi:10.1016/0002-8703(95)90088-8

[34] Y. Uchida, Y. Uchida, S. Shirai, T. Oshima, K. Shimizu, T. Tomaru, T. Sakurai and M. Kanai, "Angioscopic Detection of Pulmonary Thromboemboli," Journal of Interventional Cardiology, Vol. 23, No. 5, 2010, pp. 470-478. doi:10.1111/j.1540-8183.2010.00549.x

[35] Y. Uchida, "Percutaneous Cardioscopy of Cardiac Chambers and Valves," In: D. Zipes, Ed., Progress of Cardiology, Lea \& Febiger, Boston, 1991, pp.163-192.

[36] Y. Uchida, "Clinical Application of Percutaneous Cardioscopy for Coronary Heart Disease," In: Y. Uchida, Ed., Coronary Angioscopy, Futura Publishing Ltd., New York, 2001, pp. 181-233.

[37] Y. Uchida, F. Nakamura and T. Tomaru, "Observation of Atherosclerotic Lesions by an Intravascular Microscope in Patients with Arteriosclerosis Obliterance," American Heart Journal, Vol. 130, No. 5, 1995, pp. 1114-1117. doi:10.1016/0002-8703(95)90216-3

[38] Y. Uchida.Observation of atherosclerosis of peripheral artery by angiomicroscopy. In: Y. Uchida, Ed., Atlas of Cardioangioscopy, Medical View Ltd., Tokyo, 1995, pp. 222224.

[39] Y. Uchida, T. Sakurai, M. Kanai, S. Shirai and T. Morita, "Characterization of Coronary Fibrin Thrombus in Patients with Acute Coronary Syndrome Using Dye-Staining Angioscopy," Arteriosclerosis, Thrombosis, and Vascular Biology, Vol. 31, 2011, pp. 1452-1460. doi:10.1161/ATVBAHA.110.221671

[40] Y. Uchida, Y. Uchida, S. Kawai, R. Kanamaru, Y. Sugiyama, T. Tomaru, Y. Maezawa and N. Kameda, "Detection of Vulnerable Coronary Plaques by Color Fluorescent Angioscopy," JACC Cardiovasc Imaging, Vol. 3, No. 4, 2010, pp. 398-408. doi:10.1016/j.jemg.2009.09.030

[41] Y. Uchida and Y. Maezawa, "Molecular Imaging of Atherosclerotic Coronary Plaques by Fluorescent Angioscopy," In: B. Schaller, Ed., Molecular Imaging, InTec Open Access Publisher, New York, 2012, pp. 247-268. doi: $10.5772 / 30048$

[42] Y. Uchida and N. Hiruta, "Molecular Imaging of LowDensity Lipoprotein in Human Coronary Plaques by Color Fluorescent Angioscopy," Proceedings of 26th Annual
Meeting of Japanese Association for Cardioangioscopy, Matsuyama, 10 October 2012, p. 53.

[43] Y. Uchida, "Angioscopy Systems and Their Manipulation," In: Y. Uchida, Ed., Coronary Angioscopy, Futura Publishing Ltd., Armonk, 2001, pp. 7-24.

[44] Y. Uchida, F. Nakamura and T. Tomaru, "Rheological Significance of Tandem Lesions of the Coronary Artery," Heart Vessels, Vol. 10, No. 2, 1995, pp. 106-110. doi:10.1007/BF01744501

[45] Y. Uchida, T. Tomaru and S. Sumino, "Fiberoptic Observation of Thrombosis and Thrombolysis in Isolated $\mathrm{Hu}-$ man Coronary Artery," Amerocan Heart Journal, Vol. 112, No. 4, 1986, pp. 694-696. doi:10.1016/0002-8703(86)90462-X

[46] Y. Uchida, "Atlas of Cardioangioscopy," Medical View, Tokyo, 1995.

[47] Y. Uchida, "Clinical Classification of Atherosclerotic Coronary Plaques," In: Y. Uchida, Ed., Coronary Angioscopy, Futura Publishing Ltd., Armonk, 2001, pp. 71-82.

[48] Y. Uchida, "Peripheral Artery," In: Y. Uchida, Ed., Atlas of Cardioangioscopy, Medical View, Tokyo, 1995, pp. 190-204.

[49] Y. Uchida, J. Hirose, M. Kanai, T. Tomaru, H. Noike, S. Morizuki, T. Oshima, K. Kawamura and Y. Fujimori, "Combined Use of Angioscopy and IVUS for Evaluation of Abdominal Aortic Aneurysm before and after Open Repair with Y Graft," Proceedings of 18th Annual Meeting of Japanese Society of Endovascular Therapy, Tokyo, 20 July 2011, p. 63.

[50] Y. Uchida, "Venous Disease," In: Y. Uchida, Ed., Atlas of Cardioangioscopy, Medical View, Tokyo, 1995, pp. 206216.

[51] Y. Uchida, M. Kanai, T. Sakurai and K. Tokuhiro, "Aortoscopic Evaluation of Annuloaortic Ectasia," Proceedings of 17th Annual Meeting of Japanese Society of Endovascular Therapy, Tokyo, 5 July 2010, p. 51.

[52] Y. Uchida, "Endoscope-Guided Percutaneous Intracardiac and Intravascular Surgery," In: Y. Uchida, Ed., Atlas of Cardioangioscopy, Medical View, Tokyo, 1995, pp. 226234.

[53] Y. Uchida, Y. Fujimori, T. Tomaru, T. Oshima and J. Hirose, "Percutaneous Angioplasty of Chronic Obstructions of Peripheral Arteries by Temperature-Controlled Nd:YAG Laser System," Video Library of Japanese Society of Intravascular Therapy, 1996.

[54] Y. Uchida, Y. Maezawa, Y. Uchida, N. Hiruta and E. Shimoyama, "Molecular Imaging of Low-Density Lipoprotein in Human Coronary Plaques by Color Fluorescent Angioscopy and Microscopy," Plos One, Vol. 7, No. 11, 2012, pp. 1-9. 